\title{
Dynamics of Moving Average Rules in a Continuous-time Financial Market Model*
}

\author{
Xue-Zhong He and Min Zheng \\ School of Finance and Economics, University of Technology, Sydney \\ PO Box 123, Broadway, NSW 2007, Australia \\ tony.he1@uts.edu.au, min.zheng@uts.edu.au
}

\begin{abstract}
Within a continuous-time framework, this paper proposes a stochastic heterogeneous agent model (HAM) of financial markets with time delays to unify various moving average rules used in discrete-time HAMs. The time delay represents a memory length of a moving average rule in discrete-time HAMs. Intuitive conditions for the stability of the fundamental price of the deterministic model in terms of agents' behavior parameters and memory length are obtained. It is found that an increase in memory length not only can destabilize the market price, resulting in oscillatory market price characterized by a Hopf bifurcation, but also can stabilize an otherwise unstable market price, leading to stability switching as the memory length increases. Numerical simulations show that the stochastic model is able to characterize long deviations of the market price from its fundamental price and excess volatility and generate most of the stylized facts observed in financial markets.
\end{abstract}

Key words: Asset price, financial market behavior, heterogeneous beliefs, stochastic delay differential equations, stability, bifurcations, stylized facts.

\footnotetext{
${ }^{*}$ Current version: September 18, 2009.

Financial support from the Australian Research Council (ARC) under a Discovery Grant (DP0773776) and the University of Technology, Sydney under an ECRG (Zheng) and a research grant from the Faculty of Business (He) is gratefully acknowledged. The authors would like to thank two anonymous reviewers for providing helpful comments.
} 


\section{Introduction}

Despite the efficient market hypothesis of financial markets in the academic finance literature (see Fama, 1970), the use of technical trading rules, such as moving average rules, still seems to be widespread amongst financial market practitioners (see Allen and Taylor, 1990; Taylor and Allen, 1992). Technical analysts or "chartists", who use various technical trading rules, attempt to forecast future prices by the study of patterns of past prices and other summary statistics about security trading. Basically, they believe that shifts in supply and demand can be detected in charts of market movements.

Earlier empirical literature on stock returns finds evidences that daily, weekly and monthly returns are predictable from past returns. Pesaran and Timmermann (1994, 1995) present evidence on the predictability of excess returns on common stocks for the S\&P 500 and Dow Jones Industrial portfolios, and examine the robustness of the evidence on the predictability of U.S. stock returns. There have been various studies of the profitability of technical analysis, see Frankel and Froot (1986, 1990), Brock, Lakonishok and LeBaron (1992), Neely, Weller and Dittmar (1997), Gencay (1998) and Fernandez-Rodriguez, Gonzalez-Martel and Sosvilla-Rivero (2000).

Most of the cited research has focused on empirical studies. Recent studies, such as Lo, Mamaysky and Wang (2000), Boswijk, Griffioen and Hommes (2000) and Goldbaum (2003), have also examined explicitly the profitability of technical trading rules and the implications for market efficiency. Over the last two decades, various heterogeneous agent models (HAMs) have been developed to explain a range of market behavior. By incorporating bounded rationality and heterogeneity, HAMs have successfully explained many types of features (such as market booms and crashes, long deviations of the market price from the fundamental price), the stylized facts (such as skewness, kurtosis, volatility clustering and fat tails of returns), and various power laws (such as the long memory in return volatility) observed in financial markets. We refer the reader to Hommes (2006), LeBaron (2006) and Chiarella, Dieci and He (2009) for surveys of the recent developments in this literature.

To examine the role of moving average rules in market stability theoretically, Chiarella, He and Hommes (2006) recently propose a discrete-time HAM in which demand for traded assets has both a fundamentalist and a chartist components. The chartist demand is governed by the difference between the current price and a moving average (MA). They show analytically and numerically that the MA plays a complicated role on the stability of financial markets. In particular, when the activities of the market participants (such as the fundamentalists and the trend followers) are balanced in certain way, an increase in the memory length used in the MA can stabilize the market; otherwise, it is a source of market instability, and the interaction of the MA and market noise can lead to the tendency for the market price to take long excursions (associated with long memory length) away from the fundamental price. We also refer to Levy, Levy and Solomon (2000), Chiarella and He (2001), Zschischang and Lux (2001) and Anufriev 
and Dindo (2009) for the related discussion on the standard MA and to Chiarella, He, Hung and Zhu (2006) for weighted MA in the discrete time HAMs.

Interestingly enough, most of the HAMs in the literature are in discrete-time rather than continuous-time setup. The discrete-time setup facilities economic understanding and mathematical analysis, but it also faces some limitations when expectations of agents are formed in historical prices over different time periods. In particular, when dealing with MA rules, different lag lengths used in the MA rules lead to different dimensions of the system which need to be dealt differently, see Chiarella, He and Hommes (2006). Very often, a theoretical analysis on the impact of the memory length used in MA is difficult when the dimension of the system is higher, which is the case as the memory length used in the MA becomes large. To overcome this difficulty, this paper proposes a heterogeneous agent model of financial markets in a continuous-time framework with time delay, which represents a memory length of a moving average rule in discrete-time HAMs, to study the impact of the memory length. The financial market is consisting of a group of fundamentalists and a group of trend followers using a weighted average of historical prices as price trend. The trend followers are assumed to react to buy-sell signals generated by the difference between the current price and the price trend, which is formed as an integral with a distributed delay, representing a MA of the historical prices with an exponential decaying weights over a memory length. By incorporating random fundamental price and noisy demand from noise traders in the market, the model is described mathematically by a system of stochastic differential equations with time delay, which provides an uniform treatment on various MA rules used in the discrete-time model.

Development of deterministic delay differential equation models to characterize fluctuation of commodity prices and cyclic economic behavior has a long history, see, for example, Haldane (1932), Kalecki (1935), Goodwin (1951), Larson (1964), Howroyd and Russell (1984) and Mackey (1989). The development further leads to the studies on the effect of policy lag on macroeconomic stability, see, for example, Phillips (1954, 1957), Asada and Yoshida (2001) and Yoshida and Asada (2007). Recently, by introducing noise processes into a simple price model with a delay depended growth rate, Küchler and Platen (2007) extend the study to examine joint effect of time delay and randomness. Though there is a growing study on various market behavior and, in particular, the stylized facts, volatility clustering and long memory observed for high frequency (such as daily) returns in the discrete-time HAMs, see Lux (2004, 2009) for recent surveys, in our knowledge, using stochastic delay differential equations to study these features in financial markets is relatively new. This paper serves as the first step to extend the current HAMs from discrete-time to continuous-time within time delay framework.

By developing a stochastic HAM in continuous time with delays, this paper focuses on the impact of the behavior of heterogeneous agents and, in particular, the role of the memory length in market stability. For the underlying deterministic delay differential equation model, the stability of the fundamental price in terms of agents' behavior 
parameters and time delay are analyzed. Consistent with the results obtained in the discrete-time model in Chiarella, He and Hommes (2006), it is found that an increase in the memory length has a double edged effect on the stability, meaning that time delay can either destabilize or stabilize the market price. However, different from the discretetime model, it is also found that, depending on the behavior of the fundamentalists and trend followers, an increase in the memory length can lead to stability switching of the market price, which is new in the HAMs ${ }^{1}$. For the corresponding stochastic model, we demonstrate that the model is able to generate many market phenomena, such as market bubbles, crashes and long deviations of the market price from the fundamental price, and most of the stylized facts, including non-normality, volatility clustering, and power-law behavior of high-frequency returns, observed in financial markets.

The paper is organized as follows. We first introduce a stochastic HAM in continuous time with time delay in Section 2. In Section 3, we conduct a stability and bifurcation analysis for the underlying deterministic delay differential equation model. Section 4 provides some numerical simulation results of the stochastic model in exploring the potential of the model to generate various market behavior and the stylized facts. Section 5 concludes the paper. Proofs of some technical results are given in Appendix B.

\section{A Continuous-time HAM with Delay}

The basic idea of the modeling in this section follows closely to the current HAM framework (see, for example, Brock and Hommes, 1998; Chiarella and He, 2002, 2003; Chiarella, He and Hommes, 2006). However, instead of using a discrete-time setup, we consider a continuous-time setup. This section proposes an asset pricing model with two different types of heterogeneous traders, fundamentalists and trend followers, who trade according to fundamental analysis and technical analysis, respectively. The market price is arrived at via a market maker scenario in line with Beja and Goldman (1980), Day and Huang (1990) and Chiarella and He (2003) rather than the Walrasian scenario used in Brock and Hommes (1998) and Chiarella and He (2002). Whilst the market maker is a highly stylized account of how the market price is arrived at, it may be closer to what is going on in real markets ${ }^{2}$.

Consider a market with a risky asset (such as stock market index) and let $P(t)$ denote the price (cum dividend) per share of the risky asset at time $t$. To focus on price dynamics, we motivate the demand functions of the two different types of traders by their trading rules directly, rather than deriving the demand functions from utility maximiza-

\footnotetext{
${ }^{1}$ It should be stressed that the double edged role on the stability of time delay is not new in applied mathematical literature, in particular in mathematical biology literature, see for example MacDonald (1978) and Beretta, Bischi and Solimano (1988).

${ }^{2}$ In a recent paper by Zhu, Chiarella, He and Wang (2009), the roles of the market maker as both liquidity provider and investor are examined and it is found that, in some market conditions, the market maker has an incentive to destabilize the market.
} 
tion of their portfolio investments with both risky and risk-free assets (as for example in Brock and Hommes, 1998). The market population fractions ${ }^{3}$ of fundamentalists and chartists are respectively $\alpha$ and $1-\alpha$, where $\alpha \in[0,1]$.

Fundamentalists - The fundamentalists trade based on their estimated fundamental price. They believe that the market price $P(t)$ is mean-reverting to the fundamental price $F(t)$ that they can estimate based on various types of fundamental information, such as dividends, earnings, exports, general economic forecasts and so forth. They buy the stock when the current price $P(t)$ is below the fundamental price $F(t)$ and sell the stock when $P(t)$ is above $F(t)$. For simplicity, we assume that the demand of the fundamentalists, $Z_{f}(t)$ at time $t$, is proportional to the price deviation from the fundamental price, namely,

$$
Z_{f}(t)=\beta_{f}[F(t)-P(t)],
$$

where $\beta_{f}>0$ is a constant parameter, measuring the mean-reverting speed of the market price to the fundamental price, which may be weighted by the risk aversion coefficient of the fundamentalists. To focus the analysis on the market price, we assume that the fundamental price is given by an exogenous random process that will be specified in Section 4.

Chartists - The chartists trade based on charting signals generated from historical prices. Given the well documented momentum trading strategy in empirical literature, see for example Hirshleifer (2001), we assume that the chartists are trend followers. They believe that the future market price follows a price trend $u(t)$. When the current price is above the trend, the trend followers believe the price will rise and they like to hold a long position of the risky asset; otherwise, the trend followers will take a short position. We therefore assume that the demand of the chartists is given by

$$
Z_{c}(t)=g(P(t)-u(t))
$$

where the demand function $g$ satisfies:

$$
g^{\prime}(x)>0, \quad g^{\prime}(0)=\beta_{c}>0, \quad x g^{\prime \prime}(x)<0 \quad \text { for } x \neq 0 .
$$

The $S$-shaped demand function $g$ capturing the trend following behavior is well documented in the HAM literature (see, for example, Chiarella, Dieci and He, 2009), where the parameter $\beta_{c}$ represents the extrapolation rate of the trend followers on the future price trend when the price deviation from the trend is small. In the following discussion, we let $g(x)=\tanh \left(\beta_{c} x\right)$, which satisfies the conditions in (2.3).

Among various price trends used in practice, weighted MA rules with either finite or infinite memory length are the most popular ones. In this paper, we assume that the

\footnotetext{
${ }^{3}$ To simplify the analysis, we assume that the market fractions are constant parameters as in the market fraction model in $\mathrm{He}$ and $\mathrm{Li}$ (2008), rather than dependent variables based on some performance measure, as in Brock and Hommes (1998), or in both, as in Dieci, Foroni, Gardini and He (2006).
} 
price trend $u(t)$ of the trend followers at time $t$ is measured by an exponentially decayed weighted average of historical prices over a time interval $[t-\tau, t]$,

$$
u(t)=\frac{1}{A} \int_{t-\tau}^{t} e^{-k(t-s)} P(s) d s, \quad A=\frac{1-e^{-k \tau}}{k},
$$

where time delay $\tau \in(0, \infty)$ represents memory length of the MA, $k>0$ measures the decaying rate of the weights on the historical prices and $A$ is a normalization constant. Equation (2.4) implies that, when forming the price trend, the trend followers believe the more recent prices contain more information about the future price movement so that the weights associated to the historical prices decay exponentially with a decay rate $k$. In particular, when $k \rightarrow 0$, the weights are equal and the price trend $u(t)$ in equation (2.4) is simply given by the standard MA with equal weights,

$$
u(t)=\frac{1}{\tau} \int_{t-\tau}^{t} P(s) d s .
$$

When $k \rightarrow \infty$, all the weights go to the current price so that $u(t) \rightarrow P(t)$. In general, for $0<k<\infty$, equation (2.4) can be expressed as a delay-differential equation with time delay $\tau$

$$
d u(t)=\frac{k}{1-e^{-k \tau}}\left[P(t)-e^{-k \tau} P(t-\tau)-\left(1-e^{-k \tau}\right) u(t)\right] d t
$$

Let $\bar{P}_{\tau}(t)=e^{-k \tau} P(t-\tau)+\left(1-e^{-k \tau}\right) u(t)$ be an average price trend of the time-delay $\tau$ price and the current price trend weighted by the discount factor. From (2.6), we can see that the trend followers adjust their price trend estimation based on the deviation of the current price $P(t)$ from the average price trend. When $P(t)>\bar{P}_{\tau}(t)$, the trend followers increase their trend estimation of the risky asset. Otherwise, they decrease their trend estimation. In particular, when $\tau \rightarrow 0, \bar{P}_{\tau}(t) \rightarrow P(t)$ and $u(t) \rightarrow P(t)$, implying that the trend followers regard the current price as their price trend. When $\tau \rightarrow \infty$, the trend followers use all the historical prices (with infinite memory length) to form the price trend

$$
u(t)=\frac{1}{k} \int_{-\infty}^{t} e^{-k(t-s)} P(s) d s .
$$

Consequently, equation (2.6) becomes an ordinary differential equation

$$
d u(t)=\frac{1}{k}[P(t)-u(t)] d t .
$$

In summary, the price trend formed by the integral in (2.4) with distributed delay provides a general way to include moving average rules of either equal or decaying weights to the historical prices with either finite or infinite memory length.

Market Price via a Market Maker-Assume a net zero supply ${ }^{4}$. Then the aggregate market excess demand, weighted by the population weights of the fundamentalists and

\footnotetext{
${ }^{4}$ When the supply is positive but constant, the fundamental price needs to be adjusted to have the same price dynamics, see for example, Zhu, Chiarella, He and Wang (2009).
} 
trend followers for the risky asset, is given by $\alpha Z_{f}(t)+(1-\alpha) Z_{c}(t)$. Following Beja and Goldman (1980) and Chiarella, He and Hommes (2006), we assume that the price $P(t)$ at time $t$ is set via a market maker mechanism and is adjusted according to the aggregate excess demand, that is

$$
d P=\mu\left[\alpha Z_{F}+(1-\alpha) Z_{c}\right] d t+\sigma_{M} d W_{M}(t)
$$

where $\mu>0$ represents the speed of the price adjustment by the market maker, $W_{M}(t)$ is the standard Wiener process capturing a random excess demand process either driven by unexpected market news or noise traders, and $\sigma_{M} \geq 0$ is a constant.

A Complete Model-Based on (2.9) and the above analysis, the market price of the risky asset is determined according to the following stochastic delay differential system

$$
\left\{\begin{array}{l}
d P(t)=\mu\left[\alpha \beta_{f}(F(t)-P(t))+(1-\alpha) \tanh \left(\beta_{c}(P(t)-u(t))\right)\right] d t+\sigma_{M} d W_{M}(t), \\
d u(t)=\frac{k}{1-e^{-k \tau}}\left[P(t)-e^{-k \tau} P(t-\tau)-\left(1-e^{-k \tau}\right) u(t)\right] d t
\end{array}\right.
$$

where the fundamental price $F(t)$ is a random process which will be specified in Section 4 . The stochastic model (2.10) will be analyzed in Section 4. To understand the interaction of the deterministic dynamics and noise process, in Section 3, we study the dynamics of the corresponding deterministic model.

\section{Dynamics of the Deterministic Model}

By assuming that the fundamental price is a constant $F(t) \equiv \bar{F}$ and there is no market noise $\sigma_{M}=0$, the system (2.10) becomes a two-dimensional system of deterministic delay differential equations

$$
\left\{\begin{array}{l}
\frac{d P}{d t}=\mu\left[\alpha \beta_{f}(\bar{F}-P)+(1-\alpha) \tanh \left(\beta_{c}(P-u)\right)\right] \\
\frac{d u}{d t}=\frac{k}{1-e^{-k \tau}}\left[P(t)-e^{-k \tau} P(t-\tau)-\left(1-e^{-k \tau}\right) u(t)\right]
\end{array}\right.
$$

It is easy to see that $(\bar{P}, \bar{u})=(\bar{F}, \bar{F})$ is an equilibrium point of (3.1) where the equilibrium steady state price is given by the constant fundamental price. We therefore call $(\bar{P}, \bar{u})=$ $(\bar{F}, \bar{F})$ the fundamental steady state. In this section, we study the dynamics of the deterministic model (3.1), including the stability and bifurcation of the fundamental steady state. In general, the dynamics depend on the behavior of the fundamentalists, the trend followers, the market maker, and the time delay. It is known (see, for example, Hale and Kocak, 1991 and Gopalsamy, 1992) that the stability is characterized by the eigenvalues of the characteristic equation of the system at the steady state. For delay differential equations, the eigenvalue analysis can be complicated in general. To obtain some economic intuitions in the stability, we first consider some special cases. 


\subsection{A market of one type of agents: $\alpha=0,1$}

When $\alpha=1$ or 0 , the market consists of either the fundamentalists or the trend followers only. For $\alpha=1$, the system (3.1) is reduced to

$$
\frac{d P}{d t}=\mu \alpha \beta_{f}(\bar{F}-P(t))
$$

which represents a mean-reverting process of the market price to the fundamental price. Hence the price converges to its fundamental value eventually and therefore the fundamental steady state is globally asymptotically stable. This exhibits the stabilizing role of the fundamentalists.

For $\alpha=0$, the market consists of the trend followers only and the system (3.1) reduces to

$$
\left\{\begin{array}{l}
\frac{d P}{d t}=\mu \tanh \left(\beta_{c}(P-u)\right), \\
\frac{d u}{d t}=\frac{k}{1-e^{-k \tau}}\left[P(t)-e^{-k \tau} P(t-\tau)-\left(1-e^{-k \tau}\right) u(t)\right] .
\end{array}\right.
$$

It is easy to see that any point $(P, u)$ along the line $P=u$ is an equilibrium of $(3.2)$. This means that the system has infinite many steady states. Near the line, the solution of (3.2) with different initial values converge to different equilibria on the line $P=u$. This property is illustrated in Fig. 1. Summarizing the above analysis, we have the following result.

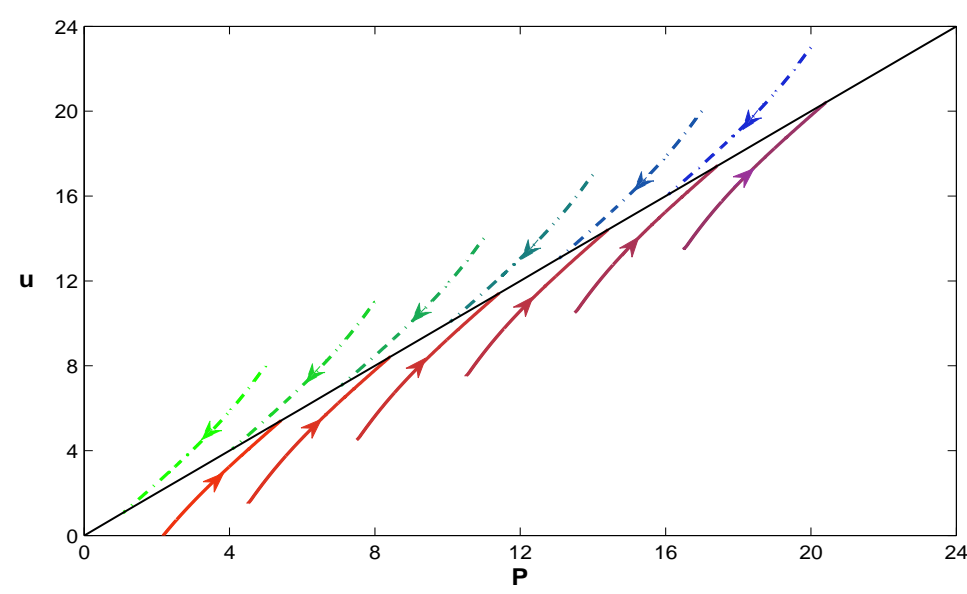

Figure 1: The local attractor line $P=u$ when $\alpha=0$. Here $\mu=1, \beta_{c}=1, k=0.5$ and $\tau=0.1$.

Proposition 3.1. (i) When the market consists of the fundamentalists only, that is, $\alpha=1$, the fundamental steady state is globally attractive; (ii) when the market consists of the trend followers only, that is, $\alpha=0$, the line $P=u$ is made up of locally stable 5 steady states.

\footnotetext{
${ }^{5}$ As pointed out by one of anonymous referees, those points $P=u$ are not attracting but only neutrally stable with respect to displacements along the line of equilibria.
} 
In the following analysis, we always assume $\alpha \in(0,1)$ unless specifically stated otherwise. In order to examine the effect of the time delay, we first consider two special cases when $\tau \rightarrow 0$ and $\tau \rightarrow \infty$ in the following subsection. We will see that the results obtained for these two special cases play an important role in our understanding of the general case $0<\tau<\infty$.

\subsection{Two special cases: $\tau \rightarrow 0$ and $\tau \rightarrow \infty$}

When $\tau \rightarrow 0$, the trend followers use the current price as their price trend $u(t) \rightarrow$ $P(t)$. Consequently, the system (3.1) is reduced to an one-dimensional system

$$
\frac{d P}{d t}=\mu \alpha \beta_{f}(\bar{F}-P)
$$

which again is a mean-reverting process. Effectively, the trend followers play no role in the market price and hence the fundamental price is globally attractive.

When $\tau \rightarrow \infty$, the price trend of the trend followers is calculated by using all historical prices. In this case, the system (3.1) becomes a two-dimensional ordinary differential system

$$
\left\{\begin{aligned}
\frac{d P}{d t} & =\mu\left[\alpha \beta_{f}(\bar{F}-P)+(1-\alpha) \tanh \left(\beta_{c}(P-u)\right)\right], \\
\frac{d u}{d t} & =k[P(t)-u(t)] .
\end{aligned}\right.
$$

The dynamics of the system (3.4) can be described by the following proposition.

Proposition 3.2. Let $\gamma_{f}=\mu \alpha \beta_{f}>0$ and $\gamma_{c}=\mu(1-\alpha) \beta_{c}>0$. System (3.4) has a unique steady state $(\bar{P}, \bar{u})=(\bar{F}, \bar{F})$ and

(i) $(\bar{F}, \bar{F})$ is globally asymptotically stable when either

$$
\gamma_{c}<k \quad \text { and } \quad \frac{k}{2}+\frac{3}{2} \gamma_{c}<\gamma_{f}
$$

or

$$
\gamma_{c}<\gamma_{f} \quad \text { and } \quad \frac{1}{2} \gamma_{f}+\frac{3}{2} \gamma_{c}<k
$$

(ii) $(\bar{F}, \bar{F})$ is locally asymptotically stable when $k+\gamma_{f}>\gamma_{c}$.

(iii) $(\bar{F}, \bar{F})$ undergoes a supercritical Hopf bifurcation when $k+\gamma_{f}=\gamma_{c}$.

(iv) $(\bar{F}, \bar{F})$ is unstable when $k+\gamma_{f}<\gamma_{c}$ and the solutions of the system converge to a stable limit cycle in the phase plane of $(P, u)$.

Proof. See Appendix B.

The sufficient conditions on the global stability in Proposition 3.2 (i) are driven by constructing suitable Lyapunov functions and, in general, they are more restrictive than the local stability condition in Proposition 3.2 (ii). This is partially due to the difficulty 
in dealing with global stability and partially due to the constructed Lyapunov functions. It is of interest to improve these conditions by constructing more suitable Lyapunov functionals. The local stability and bifurcation results of Proposition 3.2 (ii)-(iv) are illustrated in Fig. 2. In the parameter space of $\left(\gamma_{c}, k+\gamma_{f}\right)$, Fig. 2 (a) illustrates the local stability region, the Hopf bifurcation line, and the the stable limit cycle resulting from the instability and Hopf bifurcation. Figure 2 (b) plots the price bifurcation diagram with respect to the parameter $\gamma_{c}$ for given $\mu=1, \alpha=0.5, \gamma_{f}=0.5, k=0.1, \bar{F}=1$. It clearly indicates the bifurcation value at $\gamma_{c}=\gamma_{f}+k=0.6$.

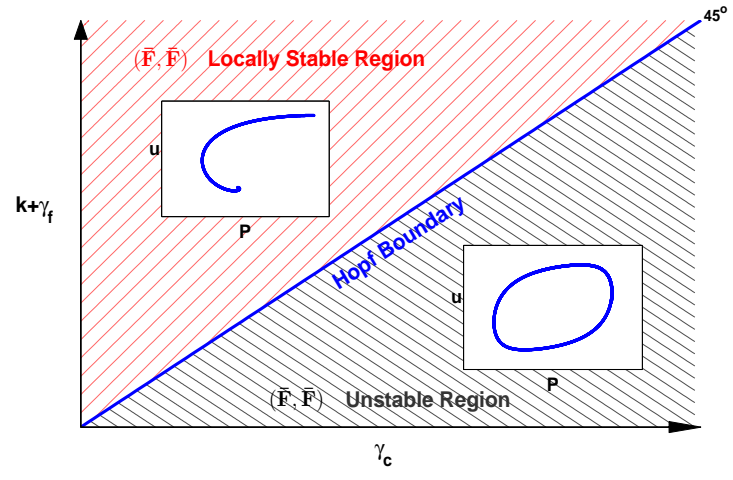

(a) Parameters region of the stability

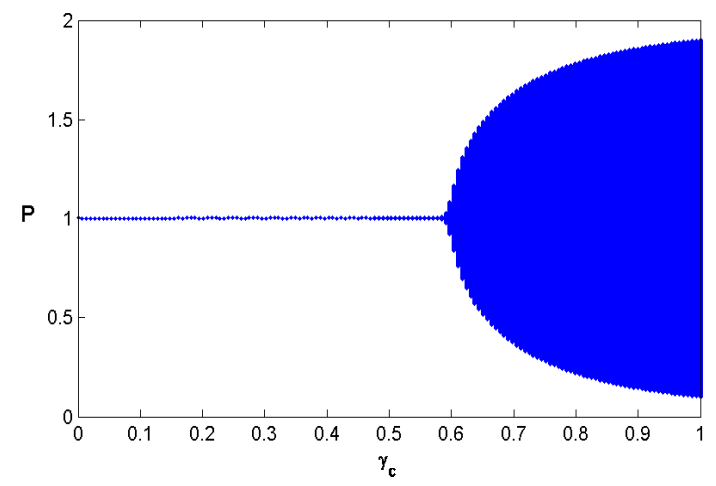

(b) Price bifurcation plot

Figure 2: (a) The regions of the stability, bifurcation and stable limit cycles in the parameter space of $\left(\gamma_{c}, k+\gamma_{f}\right)$; (b) the bifurcation diagram of the price with respect to the parameter $\gamma_{c}$ for $\mu=1, \alpha=0.5, \gamma_{f}=0.5, k=0.1, \bar{F}=1$ and $\tau \rightarrow \infty$.

The parameters $\gamma_{f}$ and $\gamma_{c}$ capture the activities of the fundamentalists and the trend followers, $\beta_{f}$ and $\beta_{c}$, weighted by the market fractions, $\alpha$ and $1-\alpha$ respectively, and the speed of the price adjustment of the market maker, $\mu$. From Proposition 3.2 and Fig. 2, we can see clearly the stabilizing role of the fundamentalists and the destabilizing role of the trend followers, which is consistent with the results in the current HAM literature within discrete-time framework. It is very interesting to see the stabilizing role of an increase in the decay rate $k$. Intuitively, the larger the decay rate $k$ is, the more weights the recent prices are given. In the limiting case $k \rightarrow \infty$, the price trend is given by the current market price and hence the role of the trend followers in the market disappears, leading to a market with the fundamentalists only. On the other hand, the smaller the decay rate $k$ is, the smoother the price trend becomes, the more sensitive to the price change the trend followers become, and the less stable the market becomes.

With the help of the discussion on the two special cases, we now examine the general case. 


\subsection{The general case: $0<\alpha<1$ and $0<\tau<\infty$}

For the general case when $0<\alpha<1$ and $0<\tau<\infty$, in this section, we first provide a result on the global stability of the fundamental steady state by constructing suitable Lyapunov functionals. By analyzing the characteristic equation of the steady state, we then examine the local stability and Hopf bifurcation of the fundamental steady state, exploring the role of the memory length and the activities of the fundamentalists and trend followers in the stability. Furthermore, we explore the possibility of regaining stability after the steady state becomes unstable as the memory length increases, leading to stability switching, a very interesting phenomenon in the continuous-time HAM but difficult to see in discrete-time HAMs. We also discuss the number of the switching. Finally, we examine the stability of two special cases, the first corresponds to the situation where the price trend is defined by the standard MA (with equal weights) and the second corresponds to the case when the activities of the fundamentalists and trend followers are perfectly balanced. Along the mathematical analysis, we also provide some economic intuitions on the complicated price dynamics of the continuous-time model.

Global Stability -Firstly, similarly to the limiting case of $\tau \rightarrow \infty$, we examine the global stability of the fundamental steady state of the delay differential equations (3.1) by Lyapunov functionals well developed in the literature, see for example, Gopalsamy (1992) and He (1998).

Proposition 3.3. The fundamental steady state of the system (3.1) is globally asymptotically stable if either

$$
\gamma_{c}<k \frac{1-3 e^{-k \tau}}{1-e^{-k \tau}} \quad \text { and } \quad \frac{3}{2} \gamma_{c}+\frac{k}{2} \frac{1+e^{-k \tau}}{1-e^{-k \tau}}<\gamma_{f},
$$

or

$$
\gamma_{c}+\frac{2 k e^{-k \tau}}{1-e^{-k \tau}}<\gamma_{f} \quad \text { and } \quad \frac{3}{2} \gamma_{c}+\frac{1}{2} \gamma_{f}<k \frac{1-2 e^{-k \tau}}{1-e^{-k \tau}} .
$$

Proof. See Appendix B.

By comparison with Proposition 3.2, we can see that the condition (3.7) (or (3.8)) implies condition (3.5) (or (3.6)). That is to say, with the increase of the time delay $\tau$ to infinity, the sufficient condition of the global stability in the delay case becomes weaker. This implies that, when the memory length is finite, the stability of the fundamental steady state becomes more involved and this becomes clear from the following analysis of the local stability of (3.1).

Local Stability and Hopf Bifurcation - The local stability of the fundamental steady state of the delay differential equation system (3.1) depends on the eigenvalues of the characteristic equation of the system at the fundamental steady state. It is known (see, for 
example, Gopalsamy, 1992) that the fundamental steady state is locally asymptotically stable if and only if all the eigenvalues $\lambda$ satisfy $\operatorname{Re}(\lambda)<0$. Let

$$
\widetilde{\tau}=\frac{1}{k} \ln \left[1+\frac{2 k \gamma_{c}}{\left(k+\gamma_{f}-\gamma_{c}\right)^{2}+2\left|k+\gamma_{f}-\gamma_{c}\right| \sqrt{k \gamma_{f}}}\right] .
$$

The following lemma on the upper bound of the time delay is helpful to the stability analysis.

Lemma 3.4. The characteristic equation of the system (3.1) at the fundamental steady state has purely imaginary roots only if $\tau \in(0, \tilde{\tau}]$.

Proof. See Appendix B.

This means that when $\tau$ is beyond $\tilde{\tau}$, there is no bifurcation and no change in the stability of the fundamental steady state. Therefore, the characteristics of (3.1) as $\tau>\widetilde{\tau}$ depend on the stability of the fundamental steady state when $\tau \rightarrow \infty$ in Proposition 3.2. The stability changes of the fundamental price happen only as the characteristic equation has a purely imaginary root $\lambda=i \omega$, which exists only if $\tau \in(0, \widetilde{\tau}]$. Define

$$
S_{n}^{ \pm}(\tau)=\tau-\frac{\theta_{ \pm}(\tau)+2 n \pi}{\omega_{ \pm}(\tau)}, \quad \tau \in(0, \widetilde{\tau}], \quad n=0,1,2, \ldots,
$$

where $\theta_{ \pm} \in[0,2 \pi)$ are the solutions of (B.9) and $\omega_{ \pm}$are the roots of equation (B.7). By the analysis in Appendix B, we know that the purely imaginary root $\lambda=i \omega$ exists if and only if there is a $\tau \in(0, \widetilde{\tau}]$ and a non-negative integer $n$ such that $S_{n}^{+}(\tau)=0$ or $S_{n}^{-}(\tau)=0$. Denote

$$
\tau_{0}=\inf \left\{\tau \in(0, \widetilde{\tau}]: \exists n \in\{0,1,2, \ldots\}, S_{n}^{+}(\tau)=0 \text { or } S_{n}^{-}(\tau)=0\right\}
$$

If the set of $\tau$ defined as above is empty, then define $\tau_{0}=\widetilde{\tau}$. Thus as $\tau \in\left(0, \tau_{0}\right)$, the fundamental steady state will keep its stability as $\tau \rightarrow 0$, that is, stable. In summary, we can obtain the following proposition.

Proposition 3.5. For (3.1), the fundamental steady state is

(i) stable for $\tau \in\left[0, \tau_{0}\right)$;

(ii) stable for $\tau>\widetilde{\tau}$ when $\gamma_{f}+k>\gamma_{c}$;

(iii) unstable for $\tau>\widetilde{\tau}$ when $\gamma_{f}+k<\gamma_{c}$.

Proposition 3.5 implies that the system can undergo a Hopf bifurcation and stability change only when $\tau \in(0, \widetilde{\tau}]$ and the stability when $\tau>\tilde{\tau}$ is completely determined by the balance between $\gamma_{f}+k$ and $\gamma_{c}$. For given decaying rate $k$, the fundamental price is locally stable when the fundamentalists are more active than the trend followers (in the sense of $\gamma_{f}+k>\gamma_{c}$ ) and the memory length is large enough, implying the stabilizing 


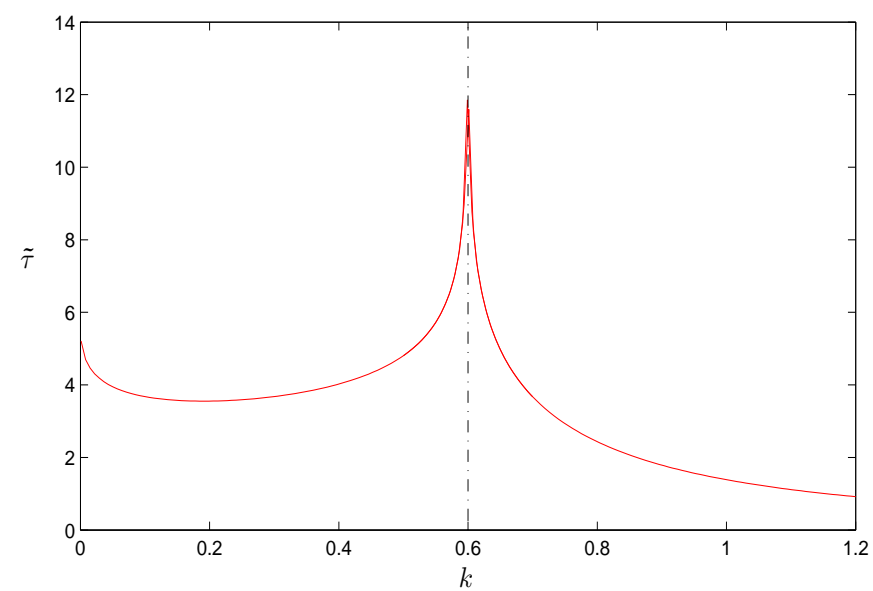

Figure 3: The upper bound $\widetilde{\tau}$ as a function of $k$. Here $\gamma_{f}=0.4$ and $\gamma_{c}=1$.

role of the increase in memory length. On the other hand, when the trend followers become more active comparing with the fundamentalists (in the sense of $\gamma_{c}>\gamma_{f}+k$ ), an increase in the memory length is destabilizing the fundamental price. Meanwhile, when the trend followers put more weights to the most recent historical prices (so that $k$ is large), the fundamental price is stabilized.

The upper bound $\widetilde{\tau}$ defined in (3.9) depends on the decay rate $k$ and, most importantly, the balance between $\gamma_{f}$ and $\gamma_{c}$. When the fundamentalists are more active (so that $\gamma_{f}>\gamma_{c}$ ), the fundamental steady state is stable for $\tau>\widetilde{\tau}$ and the upper bound $\widetilde{\tau}$ is decreasing in $k$ and satisfies that $\widetilde{\tau} \rightarrow 0$ as $k \rightarrow \infty$. In this case, increase in the memory length $\tau$ and the decay rate $k$ play stabilizing roles. However, when the trend followers are more active (so that $\gamma_{c}>\gamma_{f}$ ), there exists a critical value $k^{*}=\gamma_{c}-\gamma_{f}$ such that an increase in the memory length $\tau$ plays a different role for $k<k^{*}$ and $k>k^{*}$. For $\gamma_{f}=0.4$ and $\gamma_{c}=1$, Fig. 3 plots the upper bound $\widetilde{\tau}$ as a function of $k$. It clearly indicates that ${ }^{6}$ the function is convex for $0<k<k^{*}=0.6$ with a positive minimum value. In this case, because of $\gamma_{f}+k<\gamma_{c}$, the fundamental steady state is unstable for $\tau>\widetilde{\tau}$ and hence an increase in $\tau$ destabilizes the fundamental steady state. However, for $k>k^{*}$, the upper bound $\tilde{\tau}(k)$ is convex and decreasing, satisfying that $\widetilde{\tau} \rightarrow 0$ as $k \rightarrow \infty$. In this case, because of $\gamma_{f}+k>\gamma_{c}$, the fundamental steady state is stable for $\tau>\widetilde{\tau}$ and hence an increase in $\tau$ stabilizes the fundamental steady state. In particular, the upper bound $\widetilde{\tau} \rightarrow \infty$ when $k \rightarrow k^{*}$. This double edged role of the memory length in stability when the trend followers are more active is very interesting and has not been discussed in discrete-time HAMs. In summary, we can see that the dominance of the activities between the fundamentalists and the trend followers plays a key role on the stability of the fundamental price, while the memory length plays a secondary role.

\footnotetext{
${ }^{6}$ It can be verified that if we write $\widetilde{\tau}$ as a function of either $\gamma_{f}$ or $\gamma_{c}$, the function is convex with a positive minimum whenever $\gamma_{f}+k<\gamma_{c}$ is satisfied and convex and decreasing to zero as either $\gamma_{f} \rightarrow \infty$ or $\gamma_{c} \rightarrow 0$.
} 
Stability Switching-Proposition 3.5 indicates that the change of the stability of the fundamental price can only happen for $\tau \in\left[\tau_{0}, \tilde{\tau}\right]$. However, it does not indicate how the change occurs and the possibility of regaining the stability after the fundamental price loses its stability when the memory length increases. The following lemma is very helpful to understand this issue.

Lemma 3.6. For $\widetilde{\tau}$ defined by (3.9) and any $n=0,1,2, \ldots, S_{n}^{+}(\widetilde{\tau})=S_{n}^{-}(\widetilde{\tau})$, denoted by $S_{n}(\widetilde{\tau})$, is a decreasing series of $n$. In addition, for $n=0,1,2, \ldots$, when $k+\gamma_{f}-\gamma_{c} \neq 0$,

(i) $\lim _{\tau \rightarrow 0} S_{n}^{+}(\tau)=0$ and $\lim _{\tau \rightarrow 0} \frac{d S_{n}^{+}(\tau)}{d \tau}=-\infty$;

(ii) $\lim _{\tau \rightarrow 0} S_{n}^{-}(\tau)=c_{n}$ and $\lim _{\tau \rightarrow 0} \frac{d S_{n}^{-}(\tau)}{d \tau}=d_{n}$, where $\left\{c_{n}\right\}_{n=0}^{\infty}$ is a non-positive and decreasing series and $\left\{d_{n}\right\}_{n=0}^{\infty}$ is a negative and increasing series of $n$;

(iii) when $k+\gamma_{f}>\gamma_{c}, c_{0}=-2 \pi / \sqrt{k \gamma_{f}}<0$;

(iv) when $k+\gamma_{f}<\gamma_{c}, c_{0}=0$ and $S_{0}(\widetilde{\tau})>0$.

Proof. See Appendix B.

The properties of $S_{n}^{ \pm}(\tau)$ in Lemma 3.6, illustrated in Fig. 4(a) for $\gamma_{f}+k>\gamma_{c}$ and Fig. 4(c) for $\gamma_{f}+k<\gamma_{c}$, are very useful in helping us to understand the numbers and locations of the Hopf bifurcations. Lemma 3.6 indicates that $\left\{S_{n}(\widetilde{\tau})\right\}_{n=0}^{\infty}$ is a decreasing series of $n$ and hence $S_{0}(\widetilde{\tau})$ plays a dominating role in determining the bifurcation values. The larger the value $S_{0}(\widetilde{\tau})$ is, the more likely many functions $S_{n}^{ \pm}(\tau)$ cross zero, and the more often the stability changes.

Following Proposition 3.5, when $k+\gamma_{f}>\gamma_{c}$, the fundamental steady state is stable for $\tau \in\left[0, \tau_{0}\right) \cup(\widetilde{\tau}, \infty)$. In this case, from Lemma 3.6 (i)-(iii), we have that $S_{0}^{+}(0)=0$ and $S_{0}^{-}(0)<0$. Hence, as $\tau$ increases from 0 to $\widetilde{\tau}$, the number of the Hopf bifurcations that the system (3.1) can undergo is either 0 or even. This property is captured by the bifurcation diagrams of the market price with respect to $\tau$ in Fig. 4(b), illustrating a situation when there exist four Hopf bifurcation values for $\tau=\tau_{i}(i=1,2,3,4)$ with $0<\tau_{1}<\tau_{2}<\tau_{3}<\tau_{4}<\tilde{\tau}$. The fundamental steady state is locally stable for $\tau \in$ $\left[0, \tau_{1}\right) \cup\left(\tau_{2}, \tau_{3}\right) \cup\left(\tau_{4}, \infty\right)$ and the Hopf bifurcations lead to stable limit cycles as $\tau$ near $\tau_{i}(i=1,2,3,4)$.

When $k+\gamma_{f}<\gamma_{c}$, we know from Proposition 3.5 that the fundamental steady state is unstable for $\tau>\widetilde{\tau}$ but stable as $\tau \rightarrow 0$. In this case, from Lemma 3.6, we can see that, $S_{0}^{+}(0)=S_{0}^{-}(0)=0$ and $S_{0}(\tilde{\tau})>0$. Hence, as $\tau$ increases from 0 to $\widetilde{\tau}$, the system (3.1) must undergo at least one Hopf bifurcation and, in general, the number of the Hopf bifurcations that the system (3.1) can undergo must be odd. This property is captured by the plot of the $S_{n}^{ \pm}$as functions of $\tau$ in Fig. 4 (c) and the corresponding bifurcation diagrams of the market price with respect to $\tau$ in Fig. 4 (d), where there exist three Hopf bifurcation values for $\tau$. Summarizing the above results, we have the following corollary. 


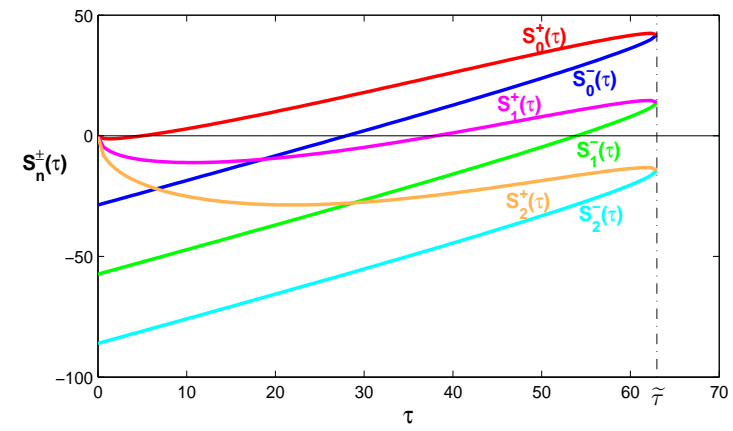

(a) Functions $S_{n}^{ \pm}$for $k=0.05, \gamma_{f}=0.96, \gamma_{c}=1$

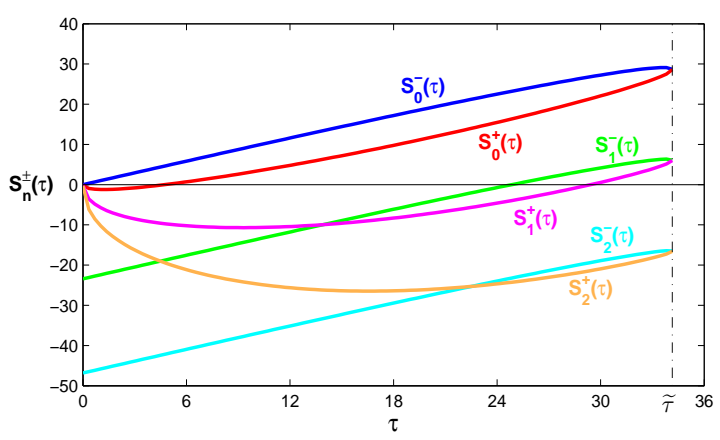

(c) Functions $S_{n}^{ \pm}$for $k=0.08, \gamma_{f}=0.9, \gamma_{c}=1$

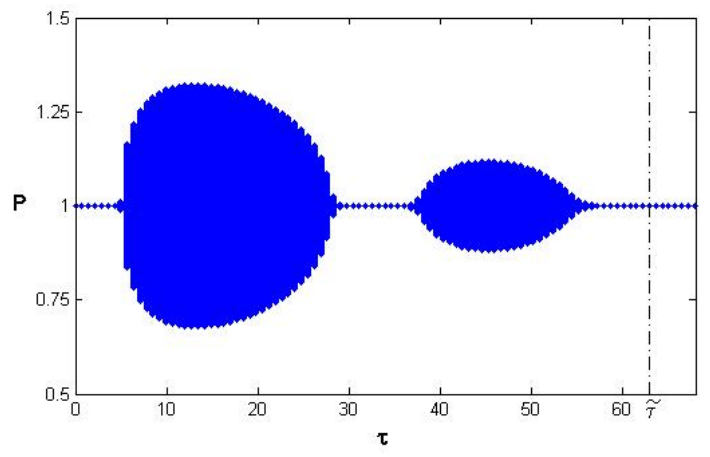

(b) Bifurcation for $k=0.05, \gamma_{f}=0.96, \gamma_{c}=1$

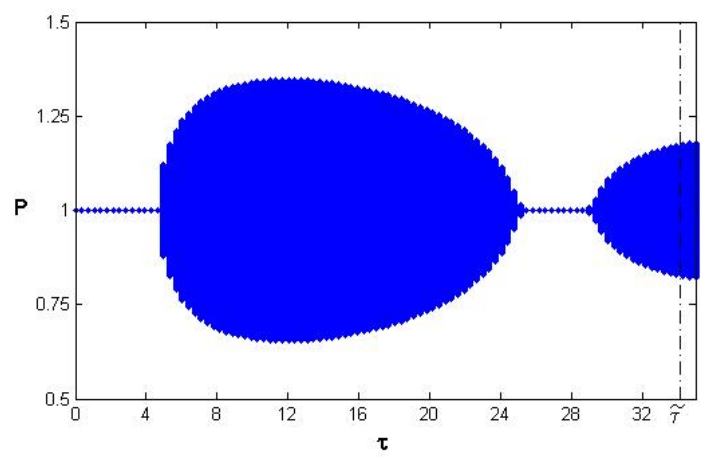

(d) Bifurcation for $k=0.08, \gamma_{f}=0.9, \gamma_{c}=1$

Figure 4: (a) and (c): the plots of $S_{n}^{ \pm}$as functions of $\tau$; (b) and (d) the bifurcation diagrams of the market price with respect to $\tau$, for $k+\gamma_{f}>\gamma_{c}$ in (a) and (b) and $k+\gamma_{f}<\gamma_{c}$ in (c) and (d). Here $\mu=1, \alpha=0.5$ and $\bar{F}=1$.

Corollary 3.7. For $\widetilde{\tau}$ defined in (3.9), the number of the Hopf bifurcations that the system (3.1) can undergo in the interval $\left[\tau_{0}, \tilde{\tau}\right]$ must be

- even (including 0) when $k+\gamma_{f}>\gamma_{c}$;

- odd when $k+\gamma_{f}<\gamma_{c}$.

The above analysis captures a very interesting phenomenon of the continuous-time HAM that is not normally observed in the discrete-time HAMs, which is the stability switching as the memory length increases. Based on the bifurcation plots in Figs 4 (b) and (d), an initial increase in $\tau$ leads to a Hopf bifurcation, which is commonly observed in the discrete-time HAMs (see for example, Chiarella, He and Hommes, 2006). However, as $\tau$ increases further, the fundamental steady state regains its stability through a second Hopf bifurcation and this kind of stability switching pattern can repeat again as $\tau$ increases further. The role of the memory length of the MA rule used by the trend followers and the stability switching pattern are difficult to be analyzed in the discrete-time HAMs due to the fact that an increase in the memory length increases the dimension of the system and the difficulty in mathematical analysis. 
For more intuitions about the properties of our model, we consider two special cases to show more about the roles of the behavior of the fundamentalists and the trend followers.

Stability under the Standard MA-In the discrete-time HAMs and learning literature, MAs with equal weights are very often used. Balasko and Royer (1996) consider local stability under a homogeneous recursive learning process formed from the past $L$ values of the state variable and show that an increase in the memory length $L$ can stabilize the system. In a HAM, Chiarella and He (2003) show that an increase in the memory length may not necessarily stabilize the system, rather generate more complicated dynamics in general. When $k \rightarrow 0$, the price trend $u(t)$ of the trend followers becomes the standard MA of the historical prices defined in (2.5). To examine the role of the memory length in the stability, we note that $S_{0}(\widetilde{\tau})$ plays a very important role in the stability, about which we have the following properties.

Lemma 3.8. As $k \rightarrow 0$,

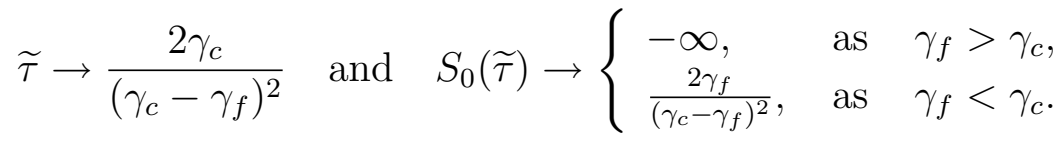

Proof. See Appendix B.

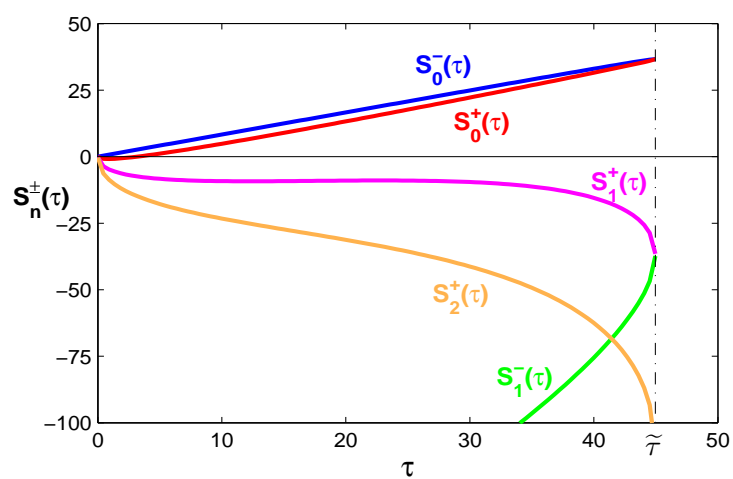

(a) Functions $S_{n}^{ \pm}$for $k=0.001, \gamma_{f}=1, \gamma_{c}=1.2$

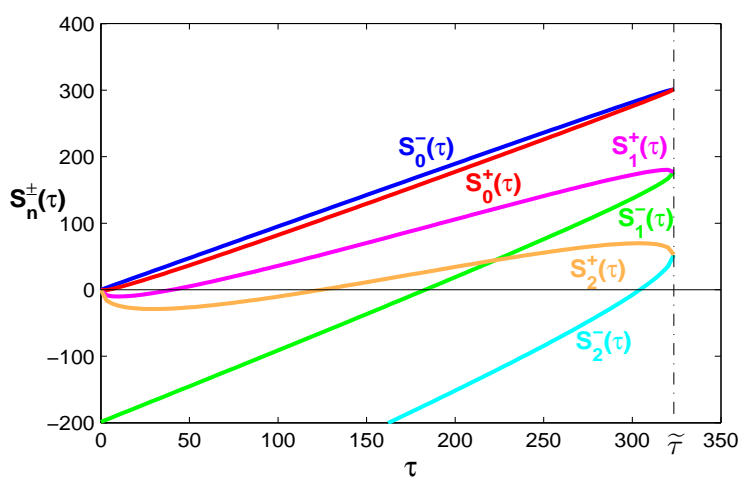

(b) Functions $S_{n}^{ \pm}$for $k=0.001, \gamma_{f}=1, \gamma_{c}=1.05$

Figure 5: Properties of functions $S_{n}^{ \pm}$when $k$ is near zero.

When $\gamma_{f}>\gamma_{c}$, that is, the activity of the fundamentalists dominates that of the trend followers, we have from Lemma 3.8 that $\lim _{k \rightarrow 0} S_{0}(\widetilde{\tau})=-\infty$. Note that $S_{n}(\widetilde{\tau}) \leq S_{0}(\widetilde{\tau})$ and $S_{n}^{ \pm}(0) \leq 0$ for any $n$. Hence there is less chance for $S_{n}^{ \pm}(\tau)$ to cross zero so that the fundamental steady state of the system (3.1) is to be more likely stable for all delay $\tau$. This observation is consistent with the stabilizing role of the fundamentalists. In comparison, when $\gamma_{c}>\gamma_{f}$, we have from Lemma 3.8 that $\lim _{k \rightarrow 0} S_{0}(\widetilde{\tau})>0$ and, in particular, $\lim _{k \rightarrow 0} S_{0}(\widetilde{\tau}) \rightarrow+\infty$ as $\gamma_{c}-\gamma_{f} \rightarrow 0$. In this case, the chance for $S_{n}^{ \pm}(\tau)$ to 
cross zero increases, leading to more complicated dynamics. For $k=0.001$ very small, this is illustrated by Fig. 5. In Fig. 5 (a), the difference $\gamma_{c}-\gamma_{f}$ is larger so that the dynamics are dominated by the trend followers, leading to a lower upper bound $\widetilde{\tau}$ for the Hopf bifurcation value and instability of the fundamental steady state for large $\tau$. In Fig. 5 (b), the difference is smaller so that neither the trend followers nor the fundamentalists dominates the market. Consequently, the upper bound $\widetilde{\tau}$ of the bifurcation value $\tau$ becomes significantly large, and the corresponding boundary value of $S_{0}(\widetilde{\tau})$, becomes very high, which increases the chance of stability switching. Also the occurrence of the Hopf bifurcation for $\tau$ is more frequent in Fig. 5 (b) than that in Fig. $5(\mathrm{a})$.

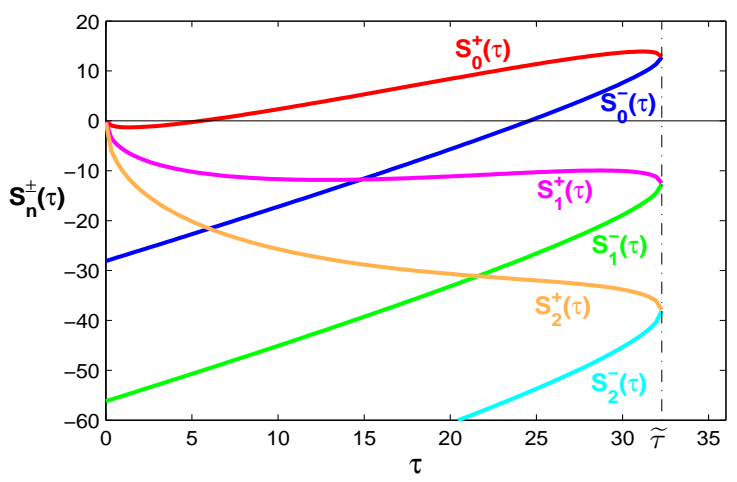

(a) Functions $S_{n}^{ \pm}$for $k=0.05, \gamma_{f}=\gamma_{c}=1$

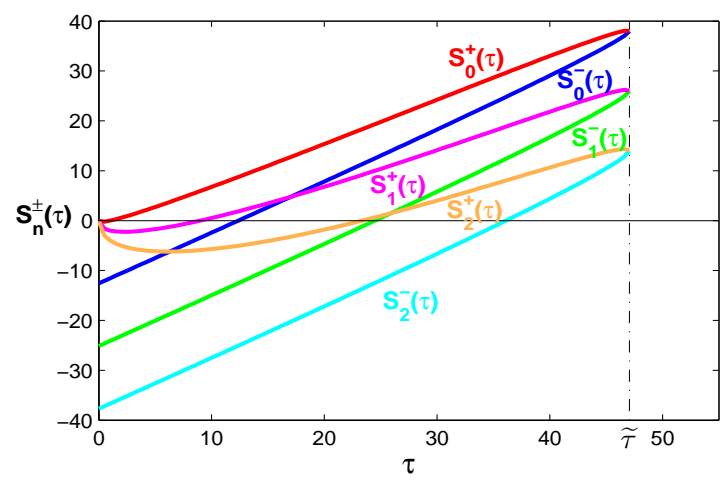

(b) Functions $S_{n}^{ \pm}$for $k=0.05, \gamma_{f}=\gamma_{c}=5$

Figure 6: Properties of functions $S_{n}^{ \pm}$when $\gamma_{f}=\gamma_{c}$.

Stability under the Balanced Activities of the Fundamentalists and Trend Followers - We have analyzed the stability when the activities of the fundamentalists and trend followers are not balanced each other (in the sense of $\gamma_{f} \neq \gamma_{c}$ ). It would be interesting to know the changes in stability when their activities are balanced. In this case, the balanced level of the activity can make a significant difference on the stability switchings. To see this, we first have the following result.

Lemma 3.9. For $\gamma_{f}=\gamma_{c}=\gamma, \lim _{\gamma \rightarrow \infty} S_{0}(\widetilde{\tau})=\infty$.

Proof. See Appendix B.

When $\gamma_{f}=\gamma_{c}=\gamma$, the fundamental steady state is stable as $\tau \rightarrow \infty$ because of $k>0$. However, it follows from Lemma 3.9 that $\lim _{\gamma \rightarrow \infty} S_{0}(\widetilde{\tau})=\infty$, which implies that surely the fundamental steady state becomes unstable for some finite time delay $\tau$ before it regains its stability when $\tau$ is large. It turns out that the level of the activity, measured by $\gamma$, plays an important role in how quickly the stability is regained. In Fig. 6 (a), the activity level is low, that is $\gamma_{f}=\gamma_{c}=1$, leading a low upper bound $\widetilde{\tau}$. This makes the fundamental steady state regain its stability quickly after the Hopf 
bifurcation. However, in Fig. 6 (b), the activity level increases, that is $\gamma_{f}=\gamma_{c}=5$, so that both the fundamentalists and the trend followers are very active. In this case, the upper bound $\widetilde{\tau}$ increases so that the fundamental steady state can only regain its stability after possible many stability switchings for possible large $\tau$.

To conclude this section, we refer to He, Li, Wei and Zheng (2009) for some further analytical results on the stability and global existence of periodic cycles resulting from the Hopf bifurcations when the fundamental steady state becomes unstable.

\section{Price Behavior of the Stochastic Model}

In this section, we conduct some numerical simulations for the stochastic model (2.10). The focus is on the interaction between the market dynamics of the deterministic model and noise processes in order to explore the potential capability of the model to generate various market behaviors, such as the long deviation of the market price from the fundamental price, and the stylized facts, including the skewness, fat tails, volatility clustering, and long memory of asset returns observed in financial markets.

To complete the stochastic model (2.10), we introduce the stochastic fundamental price process

$$
d F(t)=\frac{1}{2} \sigma_{F}^{2} F(t) d t+\sigma_{F} F(t) d W_{F}(t), \quad F(0)=\bar{F},
$$

where $\sigma_{F}>0$ represents the volatility of the fundamental return and $W_{F}(t)$ is the standard Wiener process, which is independent of the standard Wiener process for the market noise $W_{M}(t)$ introduced in (2.10). The reasons for the selection of (4.1) as the fundamental price process are two folds. The first is that the fundamental price follows a non-growing random walk process, which is in line with the market price process with no growth discussed in Section 3. The second is that the fundamental return defined by $d(\ln (F(t)))$ is a pure white noise process following the normal distribution with mean of 0 and standard deviation of $\sigma_{F}$. This ensures that any non-normality and volatility clustering of market returns that the model could generate are not carried from fundamental returns.

Firstly, we explore the joint impact of the time delay and the two noise processes on the market price dynamics. For illustration, we choose $k=0.06, \gamma_{f}=1, \gamma_{c}=1, \mu=1$, $\alpha=0.5$ and $\bar{F}=1$ so that $k+\gamma_{f}>\gamma_{c}$. For the corresponding deterministic model, we plot the phase plots of the $(u, P)$ corresponding to the underlying deterministic system (3.1) with the same initial value for three values of $\tau=3,12$ and $\tau=28$ in Figs 7 (b)-(d) corresponding to the bifurcation diagram in Fig. 7 (a). We see that the fundamental steady state is stable for both $\tau=3$ and $\tau=28$ and unstable for $\tau=12$. This implies that when the behavior of the fundamentalists dominates the market so that $\gamma_{f}+k>\gamma_{c}$, the price converges to the fundamental price for $\tau<\tau_{1}$, as illustrated in Fig. 7 (b) for $\tau=3$. As $\tau$ increases to $\tau_{1}$, the steady state is destabilized, leading to a stable limit cycle 


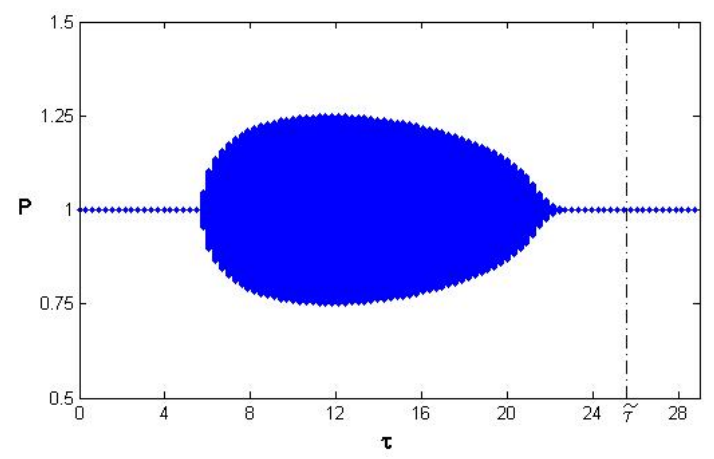

(a) Price bifurcation for $k=0.06, \gamma_{f}=1, \gamma_{c}=1$

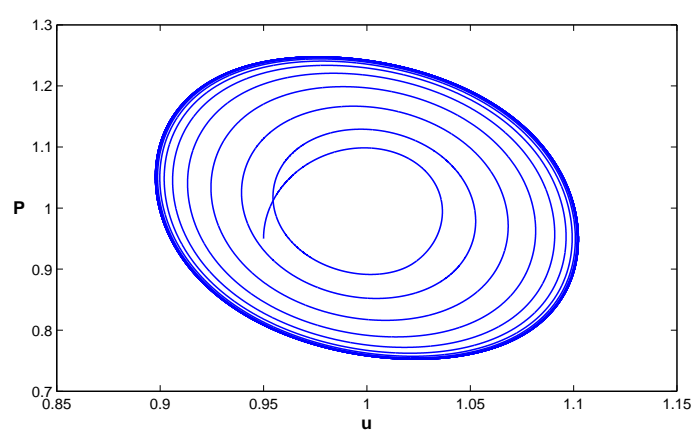

(c) Phase plot for $\tau=12$

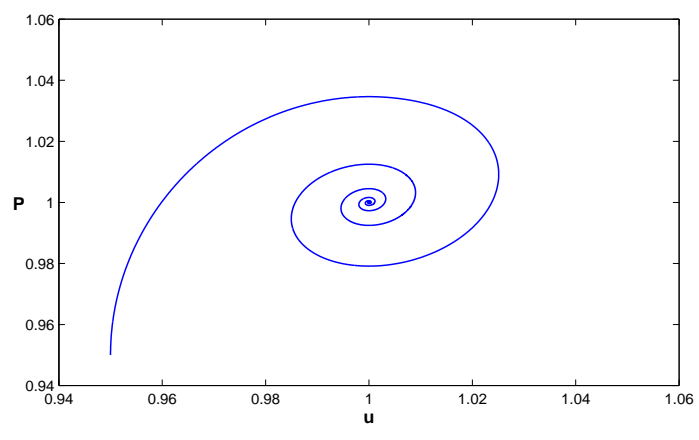

(b) Phase plot for $\tau=3$

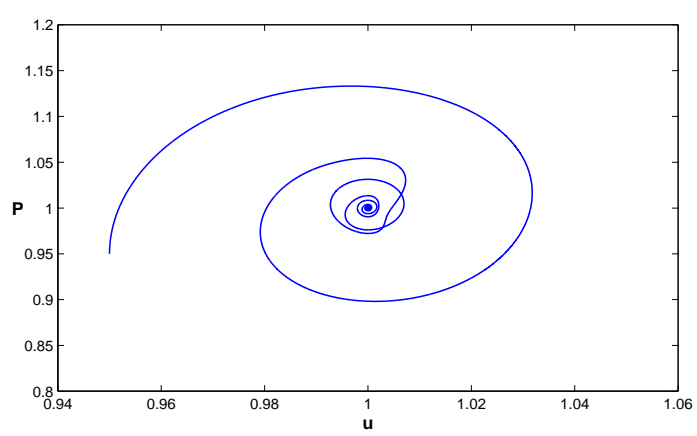

(d) Phase plot for $\tau=28$

Figure 7: (a) The bifurcation diagrams of the market price with respect to $\tau$ when $k+\gamma_{f}>\gamma_{c}$; phase plots for (b) $\tau=3$; (c) $\tau=12$; and (d) $\tau=28$. Here $k=0.06$, $\gamma_{f}=1, \gamma_{c}=1, \mu=1, \alpha=0.5$ and $\bar{F}=1$.

resulting from the Hopf bifurcation, as illustrated in Fig. 7 (c) for $\tau=12$. However, the fundamental steady state is stabilized as the trend followers increase their memory length (so that as $\tau>\tau_{2}$ ), as illustrated in Fig. 7 (d) for $\tau=28$. We also observe that, though the fundamental steady state is attractive for both $\tau=3$ and 28 , the convergence speed for $\tau=3$ is faster than that for $\tau=28$. In addition, the range of the price change for $\tau=28$ is larger than that for $\tau=3$.

For the stochastic model, we choose $\sigma_{F}=0.12$ and $\sigma_{M}=0.05$ in all the simulations in this section. With the same random draws of the fundamental price and the market noise processes, we plot the market price (the red solid line) and the fundamental price (the blue dot line) in Fig. 8 for three different values of $\tau$. For $\tau=3$, Fig. 7 (b) shows that the fundamental price of the deterministic model is stable while Fig. 8 (a) demonstrates that the market price follows the fundamental price closely for the stochastic model. For $\tau=12$, Fig. 7 (c) shows that the market price oscillates periodically around the fundamental steady state for the deterministic model while Fig. 8 (b) indicates that the market price fluctuates around the fundamental price in cyclic way for the stochastic model. For $\tau=28$, Fig. 7 (d) shows that the market price converges to the fundamental price for the deterministic model while Fig. 8 (c) illustrates the long deviation of the 
market price from the fundamental price for the stochastic model, which is underlined by the slow convergence in the deterministic model over long spans. These numerical simulations show that, together with the noises, a large delay has profound impact on the market price, though it can stabilize the fundamental steady state price in the deterministic model.
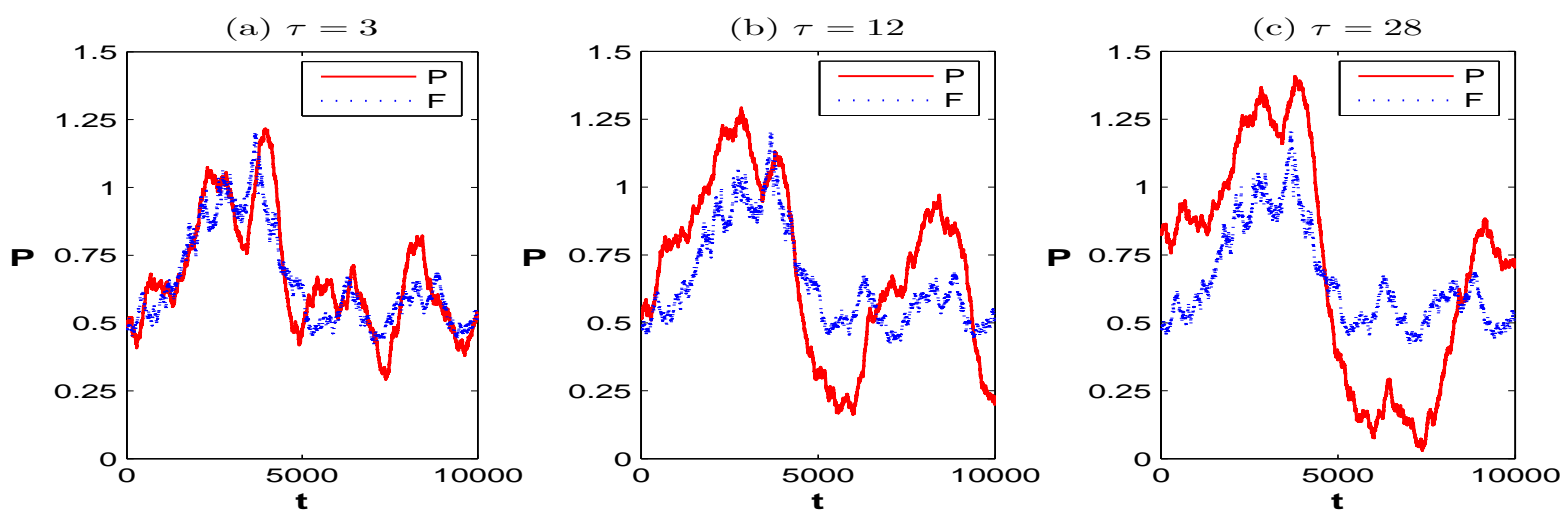

Figure 8: Time series of the fundamental price (the blue dot line) and the market price (the red solid line) for three values of (a) $\tau=3$; (b) $\tau=12$; (c) $\tau=28$. Here $k=0.06$, $\gamma_{f}=1, \gamma_{c}=1, \sigma_{F}=0.12$ and $\sigma_{M}=0.05$.

Secondly, we explore the potential of the stochastic model in generating the stylized facts for daily data observed in financial markets. In the following numerical simulations, we select $k=0.05, \gamma_{f}=1$ and $\gamma_{c}=1.1$ so that the fundamental steady state is stable for small delay but becomes unstable when the delay is large enough (because of $\gamma_{f}+k<\gamma_{c}$ ). The functions $S_{n}^{ \pm}(\tau)$ are plotted in Fig. 9 (a). We choose the time delay $\tau=1.5$ so that the fundamental steady state is locally asymptotically stable, as illustrated by the phase plot of the price trend $u(t)$ and the price $P(t)$ in Fig. $9(\mathrm{~b})$.

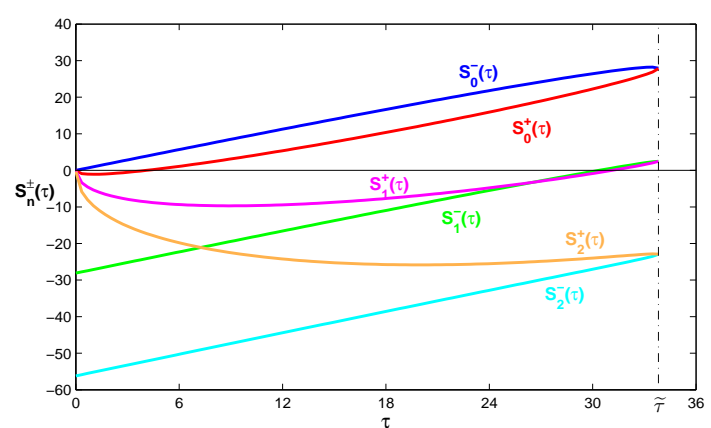

(a) Functions $S_{n}^{ \pm}(\tau)$

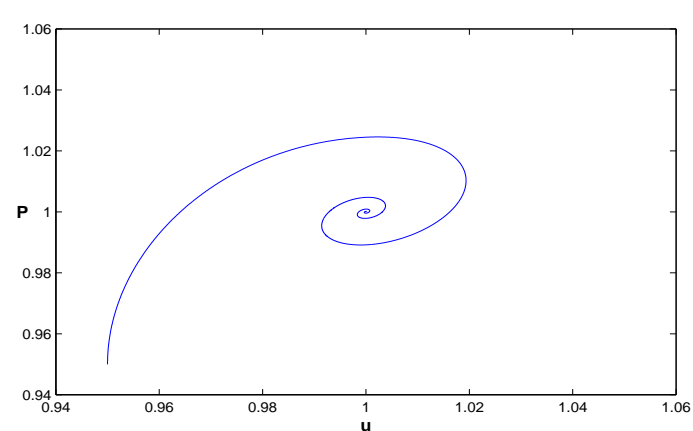

(b) Phase plot of the $(u(t), P(t))$.

Figure 9: (a) The functions $S_{n}^{ \pm}(\tau)$ and (b) the phase plot of the price trend $u(t)$ and price $P(t)$ for $\tau=1.5$, here $k=0.05, \gamma_{f}=1, \gamma_{c}=1.1$ and $\bar{F}=1$.

For the stochastic model with both noise processes, Fig. 10 represents the results of a typical simulation where the time step is one day. Figure 10 (a) shows that the 


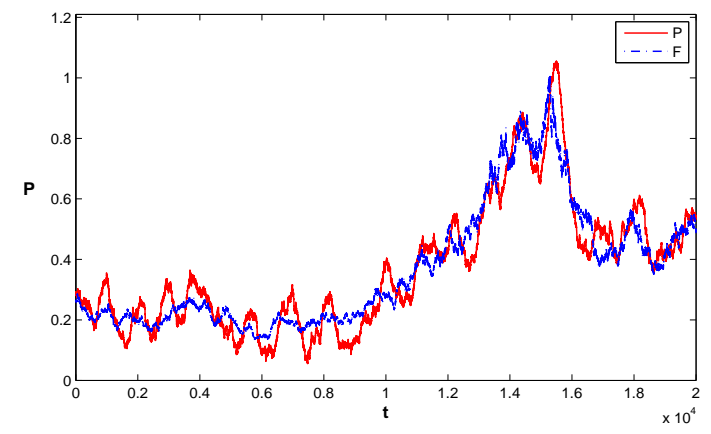

(a) The market price and the fundamental price

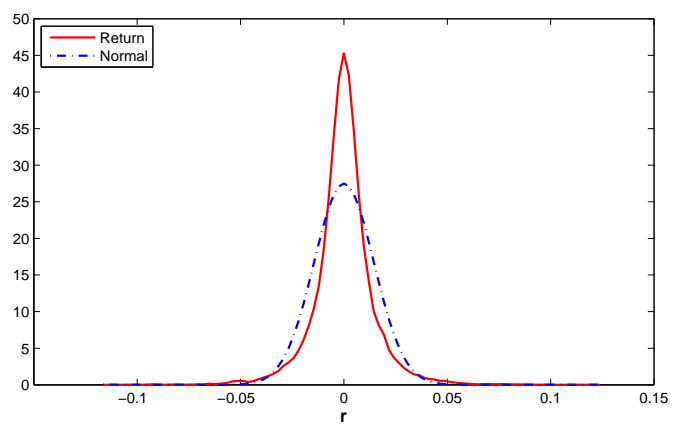

(c) The density of the market return

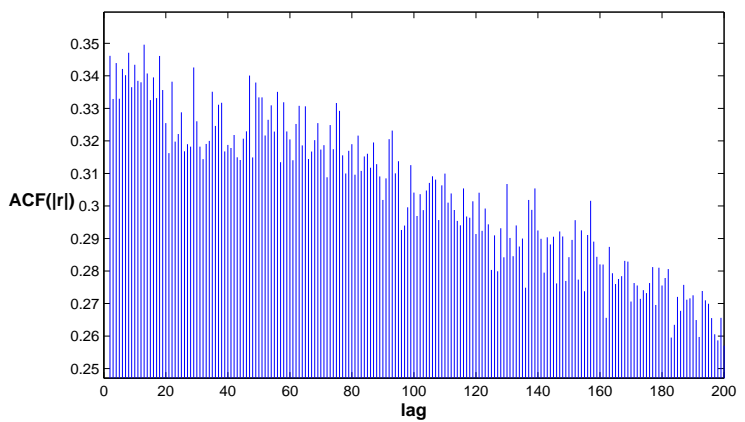

(e) The ACs of the absolute return

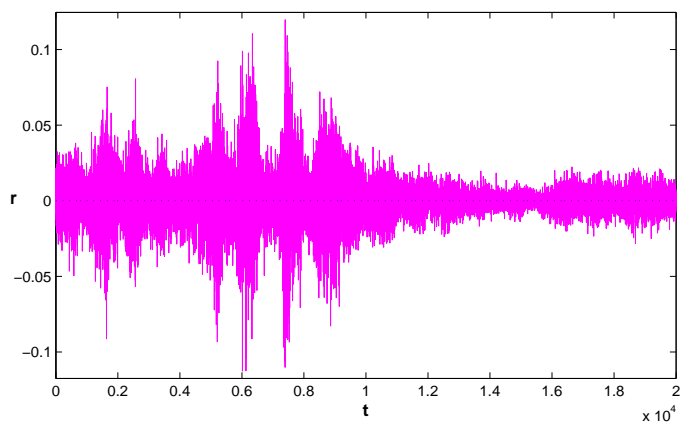

(b) The market return $(r)$

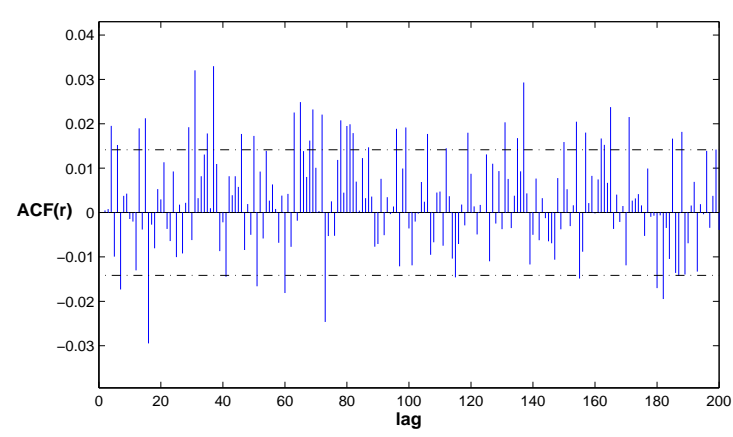

(d) The ACs of the market return

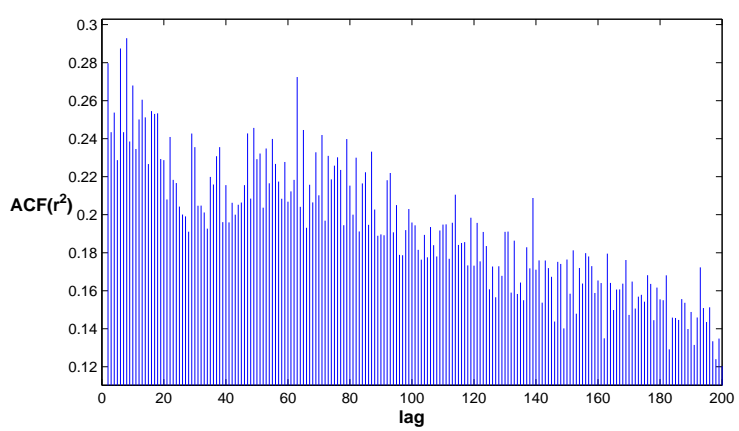

(f) The ACs of the squared return

Figure 10: The time series of (a) the market price (red solid line) and the fundamental price (blue dash-dot line) and (b) the market return; (c) the density distribution of the market returns; the ACs of (d) the market returns, (e) the absolute returns, and (f) the squared returns. Here $\sigma_{F}=0.12$ and $\sigma_{M}=0.05$. 
market price (the red solid line) follows the fundamental price (the blue dash-dot line) in general, but companied with large deviations from time to time. The returns of the market prices in Fig. 10 (b) show significant volatility clustering. Comparing to the corresponding normal distribution, the return density distribution in Fig. 10 (c) displays high kurtosis. The returns show almost insignificant autocorrelations (ACs) in Fig. 10 (d), but the ACs for the absolute returns and the squared returns in Figs 10 (e)-(f) are significantly with strong decaying patterns as time lag increases. The result demonstrates that the stochastic model has a great potential to generate most of the stylized facts observed in financial markets.

We may argue that the above features of the stochastic model is a joint outcome of the interaction of the nonlinear HAM and the two stochastic processes. In Appendix A, with the same random seeds, we report the simulation results when there is only one stochastic process involved. In Fig. 11, there is no market noise process and the fundamental price is the only stochastic process. The time series, return density distribution, and the ACs of the return, the absolute return and the squared return do not replicate these stylized facts demonstrated in Fig. 10. Alternatively, in Fig. 12, the market noise process is the only stochastic process. It shows that the return is basically described by a white noise process. Both Figs 11 and 12 indicate that the potential of the model in generating the stylized facts is not due to either one of the two stochastic processes, rather than to both processes. The findings of the continuous-time HAM model on the potential of generating the stylized facts in this paper are very similar to that of the discrete-time HAM model discussed in He and Li (2007).

\section{Conclusion and Discussion}

The continuous-time HAM with time delay proposed in this paper provides a unified treatment to the discrete-time HAMs involved expectations that are formed by using historical information, such as weighted MA rules. However, the correspondence between the behavior of high dimensional discrete-time models and infinite dimensional continuous-time models with delays such as (3.1) may be severely limited. In particular, the stability switching as the memory length increases is apparently little known for the current discrete-time HAM literature. It is clear from the present work and the HAM literature (see, for example, Chiarella, He and Hommes, 2006) that, when agents use lagged information such as price to form the expectation, an increase in the memory length can either stabilize or destabilize the system in general. The analysis presented in this paper shows that, under certain circumstance, such stabilizing and destabilizing effect can switch (possibly many times) as the memory length increases. Numerical simulations of the stochastic model show that the stability switching as the memory length increases in the deterministic model can generate long deviation of the market price from the fundamental price. It also demonstrates the potential of the stochastic model 
in generating most of the stylized facts observed in financial markets.

In order to make the model parsimonious and focus on the memory length effect, we consider a very simple financial market with heterogeneous agents. It is of interest to extend the model in a number of directions. First, the demand functions of the heterogeneous agents in the paper are assumed based on agents' behavior rather than on utility maximization in the standard finance theory. Justification and variation of the behavior demand functions using utility maximization are of interest. Second, the market clearing mechanism used in this paper is via a market maker who provides liquidity to the market. It is well known that (see, for example, Bradfield, 1979) the market maker acts not only as a liquidity provider but also as an active investor by managing the market maker inventory in order to maximize the profit (see, for example, Zhu, Chiarella, He and Wang, 2009). To incorporate the market maker inventory into the present framework and to examine the market impact of the time delay are of interest. In addition, the analysis of the stochastic model in the paper is based on some numerical simulations. Also, we only consider both the market and fundamental price processes without growth. A systematic study on the interaction of the nonlinearity of the model and different forms of the stochastic processes is desirable in order to understand the mechanism of generating the stylized facts. In future research it will be useful to understand those issues. 


\section{Appendix A. Results of stochastic simulations with one noise process}

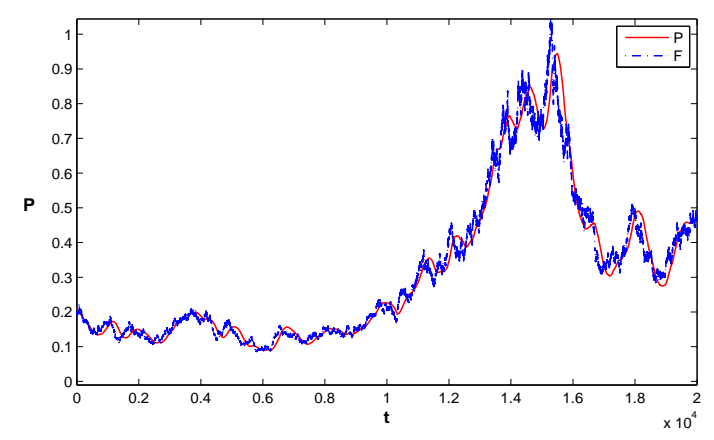

(a) The market price and the fundamental price

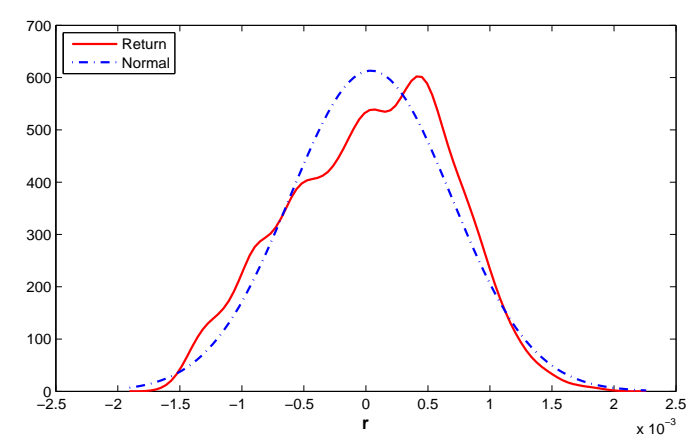

(c) The density of the market return

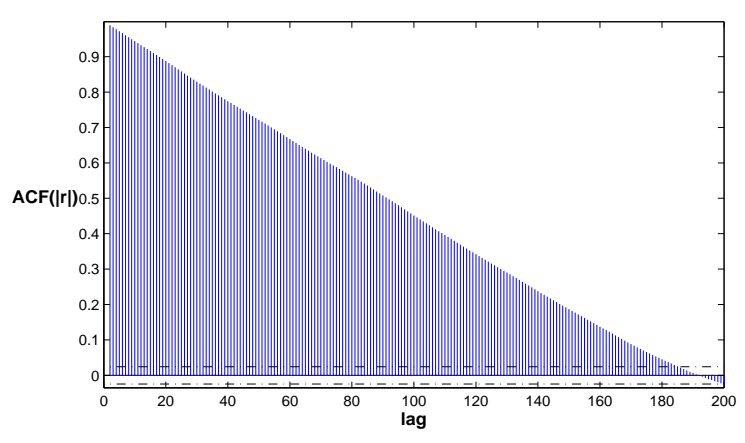

(e) The ACs of the absolute return

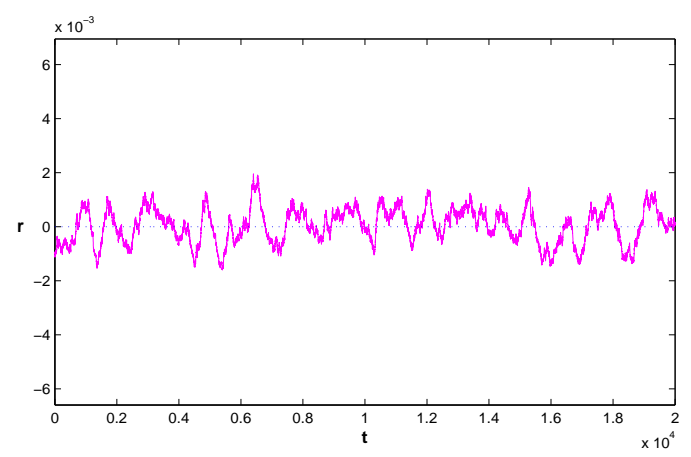

(b) The market $\operatorname{return}(r)$

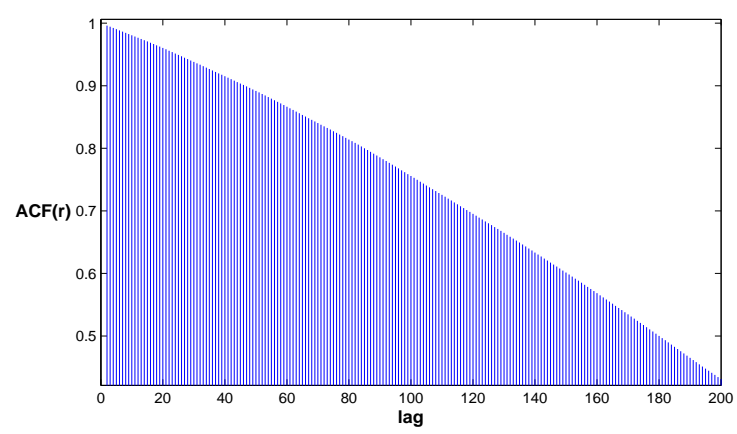

(d) The ACs of the market return

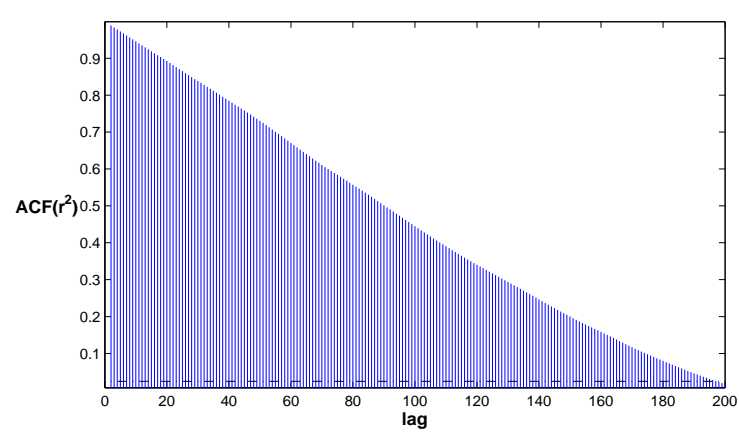

(f) The ACs of the squared return

Figure 11: The time series of (a) the market price (red solid line) and the fundamental price (blue dash-dot line) and (b) the market return; (c) the density distribution of the market returns; the ACs of (d) the market returns, (e) the absolute returns, and (f) the squared returns. Here $\sigma_{F}=0.12$ and $\sigma_{M}=0$. 


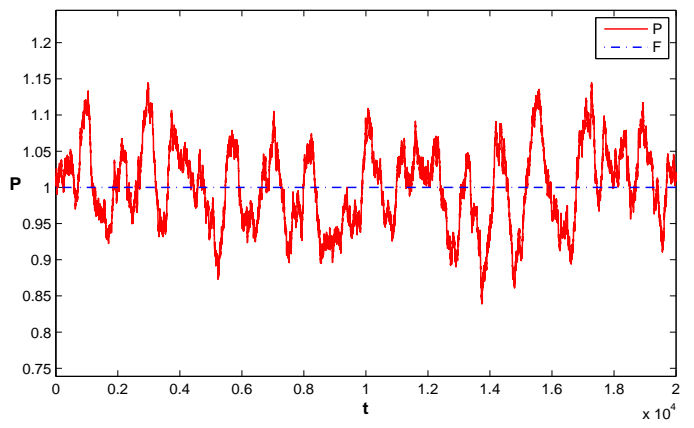

(a) The market price and the fundamental price

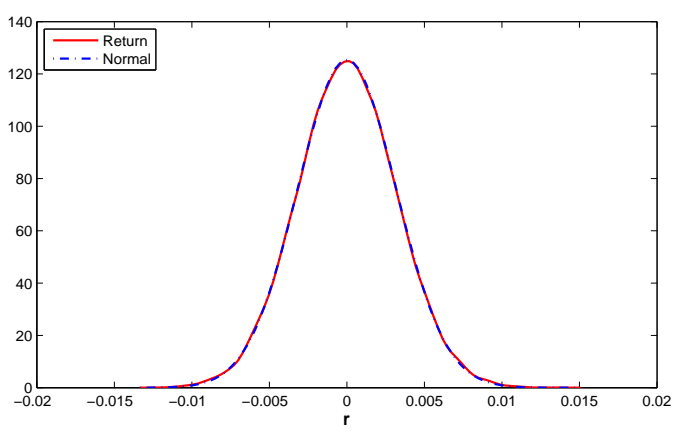

(c) The density of the market return

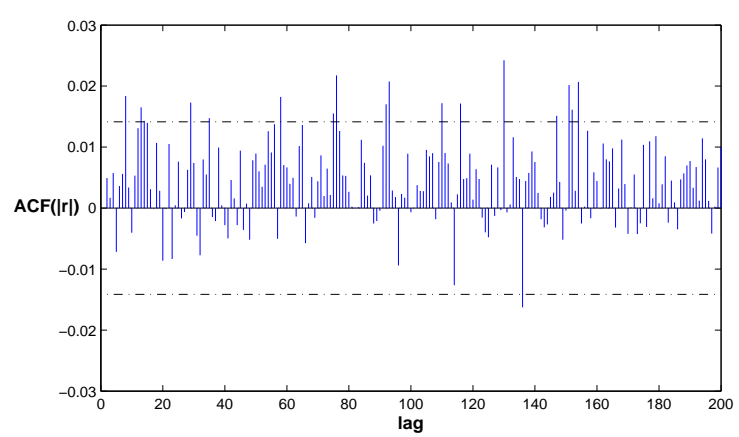

(e) The ACs of the absolute return

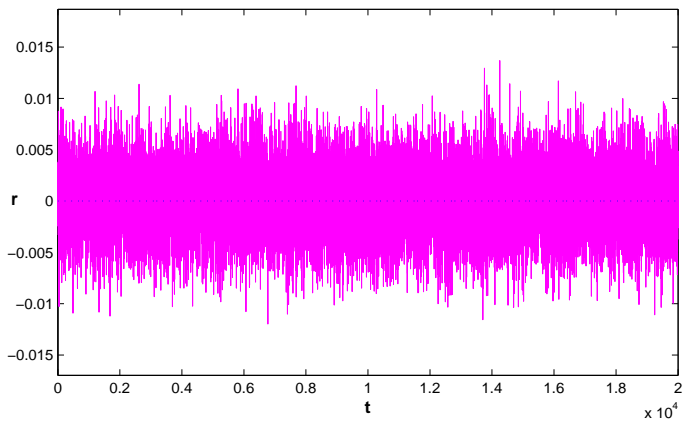

(b) The market $\operatorname{return}(r)$

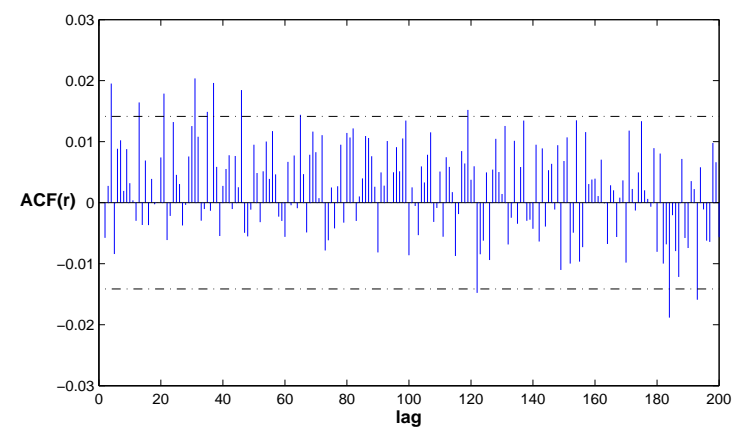

(d) The ACs of the market return

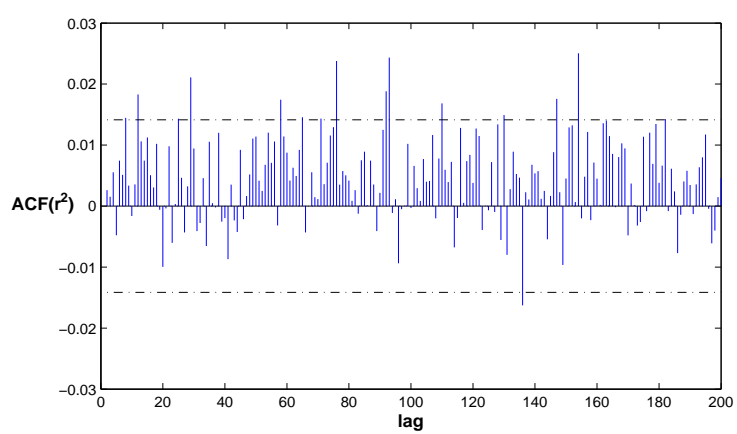

(f) The ACs of the squared return

Figure 12: The time series of (a) the market price (red solid line) and the fundamental price (blue dash-dot line) and (b) the market return; (c) the density distribution of the market returns; the ACs of (d) the market returns, (e) the absolute returns, and (f) the squared returns. Here $\sigma_{F}=0$ and $\sigma_{M}=0.05$. 


\section{Appendix B. Proofs of the main results}

Proof of Proposition 3.2: We first prove the global stability results. Let $x(t)=P(t)-\bar{F}$ and $y(t)=u(t)-\bar{F}$. Then the system (3.4) becomes

$$
\begin{aligned}
\frac{d x(t)}{d t} & =-\gamma_{f} x(t)+\mu(1-\alpha) \tanh \left(\beta_{c}(x(t)-y(t))\right), \\
\frac{d y(t)}{d t} & =k[x(t)-y(t)] .
\end{aligned}
$$

Define a Lyapunov function $V_{1}(x(t), y(t))=\frac{1}{2}\left[x^{2}(t)+y^{2}(t)\right]$. Then one can verify that, under condition (3.5),

$$
\frac{d V_{1}}{d t} \leq\left(-\gamma_{f}+\frac{3}{2} \gamma_{c}+\frac{k}{2}\right) x^{2}(t)+\frac{1}{2}\left[\gamma_{c}-k\right] \gamma_{c} y^{2}(t)<0
$$

Applying the Lyapunov Theorem, this leads to the global stability of the fundamental steady state.

To show the global stability under the condition (3.6), we change the variables from $(x(t), y(t))$ to $(x(t), z(t))$ where $z(t)=y(t)-x(t)$ and define the Lyapunov function by $V_{2}(x(t), z(t))=\frac{1}{2}\left[x^{2}(t)+z^{2}(t)\right]$. Then the result follows from

$$
\frac{d V_{2}}{d t} \leq\left(\frac{\gamma_{c}}{2}-\frac{\gamma_{f}}{2}\right) x^{2}+\left(\frac{3 \gamma_{c}}{2}+\frac{\gamma_{f}}{2}-k\right) z^{2}<0
$$

On the local stability, the characteristic equation of the system (3.4) at the fundamental steady state $(\bar{F}, \bar{F})$ is

$$
\lambda^{2}+\left(k+\gamma_{f}-\gamma_{c}\right) \lambda+k \gamma_{f}=0
$$

Note that $k>0$ and $\gamma_{f}>0$. To ensure that all the solutions of (B.1) have negative real parts, the necessary and sufficient condition is

$$
k+\gamma_{f}-\gamma_{c}>0
$$

When $k+\gamma_{f}-\gamma_{c}=0$, (B.1) has a pair of conjugate complex roots. By the normal form theory, at $\gamma_{c}=k+\gamma_{f}$, we can get the first Lyapunov coefficient of (3.4)

$$
\ell_{1}=-\frac{\left(k+\gamma_{f}\right)^{3} \sqrt{k \gamma_{f}}}{2 \mu^{2} k^{2}(1-\alpha)^{2}}<0
$$

corresponding to $(\bar{F}, \bar{F})$. Thus, $(\bar{F}, \bar{F})$ undergoes a supercritical Hopf bifurcation (see Kuznetsov, 2004) at $\gamma_{c}=k+\gamma_{f}$.

Proof of Proposition 3.3: $\quad$ Let $x(t)=P(t)-\bar{F}$ and $y(t)=u(t)-\bar{F}$. Then the system (3.1) becomes

$$
\begin{aligned}
& \frac{d x(t)}{d t}=-\gamma_{f} x(t)+\mu(1-\alpha) \tanh \left(\beta_{c}(x(t)-y(t))\right), \\
& \frac{d y(t)}{d t}=\frac{k}{1-e^{-k \tau}}\left[x(t)-e^{-k \tau} x(t-\tau)-\left(1-e^{-k \tau}\right) y(t)\right] .
\end{aligned}
$$


Define a Lyapunov functional

$$
V_{1}(x(t), y(t), x(t-\tau))=\frac{1}{2}\left[x^{2}(t)+y^{2}(t)\right]+\frac{1}{2} \frac{k e^{-k \tau}}{1-e^{-k \tau}} \int_{t-\tau}^{t} x^{2}(s) d s .
$$

Then one can verify that

$$
\begin{aligned}
\frac{d V_{1}}{d t} \leq & {\left[-\gamma_{f}+\frac{3}{2} \gamma_{c}+\frac{1}{2} \frac{k\left(1+e^{-k \tau}\right)}{1-e^{-k \tau}}\right] x^{2}(t) } \\
& +\left[\frac{1}{2} \gamma_{c}+\frac{k}{1-e^{-k \tau}}\left(\frac{1}{2}\left(1+e^{-k \tau}\right)-\left(1-e^{-k \tau}\right)\right)\right] y^{2}(t),
\end{aligned}
$$

Applying the Lyapunov Theorem (see Gopalsamy, 1992), we can show the global stability of the fundamental steady state under the condition (3.7).

To show the global stability under the condition (3.8), we change the variables from $(x(t), y(t))$ to $(x(t), z(t))$ where $z(t)=y(t)-x(t)$ and define the Lyapunov function by

$$
V_{2}(x(t), z(t), x(t-\tau))=\frac{1}{2}\left[x^{2}(t)+z^{2}(t)\right]+\frac{1}{2} \frac{k e^{-k \tau}}{1-e^{-k \tau}} \int_{t-\tau}^{t} x^{2}(s) d s .
$$

Then the result follows from

$$
\frac{d V_{2}}{d t} \leq\left[\frac{\gamma_{c}}{2}-\frac{\gamma_{f}}{2}+\frac{k e^{-k \tau}}{1-e^{-k \tau}}\right] x^{2}+\left[\frac{3 \gamma_{c}}{2}+\frac{\gamma_{f}}{2}+\frac{k}{1-e^{-k \tau}}\left(e^{-k \tau}-\left(1-e^{-k \tau}\right)\right)\right] z^{2}<0 .
$$

Proof of Lemma 3.4: The local stability of the fundamental steady state of the delay differential equation system (3.1) depends on the eigenvalue $\lambda$ of the characteristic equation of the system at the fundamental steady state

$$
p(\lambda, \tau)+q(\tau) e^{-\lambda \tau}=0,
$$

which is a transcendental equation, where

$$
p(\lambda, \tau)=\lambda^{2}+\left(k+\gamma_{f}-\gamma_{c}\right) \lambda+k \gamma_{f}-k \gamma_{c}+\frac{k \gamma_{c}}{1-e^{-k \tau}}, \quad q(\tau)=-\frac{k \gamma_{c} e^{-k \tau}}{1-e^{-k \tau}} .
$$

The fundamental steady state is locally asymptotically stable if and only if all the eigenvalues $\lambda$ of (B.3) satisfy $\operatorname{Re}(\lambda)<0$. As $k \gamma_{f}>0$, it is easy to see that $\lambda=0$ is not a root of equation (B.3). Thus the steady state becomes unstable when the characteristic equation (B.3) has purely imaginary roots. The bifurcation theory implies that, in this case, complex phenomena result from a Hopf bifurcation ${ }^{7}$.

Let $\lambda=i \omega(\omega>0)$ be a root of (B.3). Substituting it into (B.3), then the real and imaginary parts satisfy

$$
\omega^{2}-k \gamma_{f}-\frac{k \gamma_{c} e^{-k \tau}(1-\cos (\omega \tau))}{1-e^{-k \tau}}=0, \quad \omega\left(k+\gamma_{f}-\gamma_{c}\right)+\frac{k \gamma_{c} e^{-k \tau} \sin (\omega \tau)}{1-e^{-k \tau}}=0,
$$

\footnotetext{
${ }^{7}$ We refer the reader to Beretta and Kuang (2002) for a bifurcation analysis of delay differential equations by establishing a geometrical criterion on the existence of purely imaginary roots of a characteristic equation with delay-dependant coefficients.
} 
from which, we obtain the trigonometric function values

$$
\sin (\omega \tau)=\frac{-\omega\left(1-e^{-k \tau}\right)\left(k+\gamma_{f}-\gamma_{c}\right)}{k \gamma_{c} e^{-k \tau}}, \quad \cos (\omega \tau)=1-\frac{\left(1-e^{-k \tau}\right)\left(\omega^{2}-k \gamma_{f}\right)}{k \gamma_{c} e^{-k \tau}}
$$

It then follows from $\sin ^{2}(\omega \tau)+\cos ^{2}(\omega \tau)=1$ that $\omega$ must satisfy

$$
\omega^{4}+a_{1} \omega^{2}+a_{2}=0
$$

where

$$
a_{1}=k^{2}+\gamma_{f}^{2}+\gamma_{c}^{2}-2 \gamma_{f} \gamma_{c}-\frac{2 k \gamma_{c}}{1-e^{-k \tau}}, \quad a_{2}=k^{2} \gamma_{f}^{2}+\frac{2 k^{2} \gamma_{c} \gamma_{f} e^{-k \tau}}{1-e^{-k \tau}}
$$

Equation (B.7) has positive roots only if $\tau$ is defined in $(0, \widetilde{\tau}$.

Proof of Lemma 3.6: Equation (B.3) has the purely imaginary root $\lambda=i \omega(\omega>0)$ only if $\tau \leq \widetilde{\tau}$ and

$$
\tau=\frac{\theta+2 n \pi}{\omega}, \quad n=0,1,2, \ldots,
$$

where $\omega$ is the root of equation (B.7) and $\theta \in[0,2 \pi)$ satisfies

$$
\sin (\theta)=\frac{-\omega\left(1-e^{-k \tau}\right)\left(k+\gamma_{f}-\gamma_{c}\right)}{k \gamma_{c} e^{-k \tau}}, \quad \cos (\theta)=1-\frac{\left(1-e^{-k \tau}\right)\left(\omega^{2}-k \gamma_{f}\right)}{k \gamma_{c} e^{-k \tau}} .
$$

Note that for fixed $\tau \in(0, \widetilde{\tau}],\left(\right.$ B.7) has two positive roots $\omega_{+}(\tau)$ and $\omega_{-}(\tau)$ with $\omega_{+}(\tau) \geq \omega_{-}(\tau)$. We denote

$$
S_{n}^{+}(\tau)=\tau-\frac{\theta_{+}(\tau)+2 n \pi}{\omega_{+}(\tau)}, \quad S_{n}^{-}(\tau)=\tau-\frac{\theta_{-}(\tau)+2 n \pi}{\omega_{-}(\tau)}, \quad n=0,1,2, \ldots,
$$

where $\theta_{ \pm}$are the solutions of equations (B.9) corresponding to $\omega_{ \pm}$. Hence, the imaginary root $\lambda=i \omega$ of equation (B.3) exists if and only if there is a $\tau \in(0, \widetilde{\tau}]$ and a non-negative integer $n$ such that $S_{n}^{+}(\tau)=0$ or $S_{n}^{-}(\tau)=0$.

For the properties of $S_{n}^{ \pm}(\tau)$, we only provide the proof of the case $k+\gamma_{f}-\gamma_{c}>0$ here and the proof of the case $k+\gamma_{f}-\gamma_{c}<0$ is similar. Note that, for $k+\gamma_{f}-\gamma_{c}>0$,

$$
\theta_{ \pm}=2 \pi-\arccos \left(1-\frac{\left(1-e^{-k \tau}\right)\left(\omega_{ \pm}^{2}-k \gamma_{f}\right)}{k \gamma_{c} e^{-k \tau}}\right) \in(0,2 \pi) \text { and } \omega_{ \pm}^{2}=-\frac{1}{2} a_{1} \pm \frac{a_{1}^{2}-4 a_{2}}{2}
$$

When $\tau \rightarrow 0, \omega_{+} \rightarrow+\infty$ and $\omega_{-} \rightarrow k \gamma_{f}$, leading to $\theta_{-} \rightarrow 2 \pi$. Furthermore, $\lim _{\tau \rightarrow 0} S_{0}^{+}=0$ and $\lim _{\tau \rightarrow 0} S_{0}^{+}=-2 \pi / \sqrt{k \gamma_{f}}$. Let $x=1-\exp (-k \tau)$, then

$$
\begin{aligned}
\frac{d S_{n}^{ \pm}}{d x}=\frac{1}{k(1-x)} & -\frac{\omega_{ \pm}^{2}-k \gamma_{f}}{x^{2} \omega_{ \pm}^{2} \sqrt{a_{1}^{2}-4 a_{2}}}\left[ \pm \frac{2 k \gamma_{c}}{k+\gamma_{f}-\gamma_{c}}\right. \\
& \left.-\frac{x \sqrt{a_{1}^{2}-4 a_{2}}}{(x-1)\left(k+\gamma_{f}-\gamma_{c}\right)} \pm \frac{k \gamma_{c}\left(\theta_{ \pm}+2 n \pi\right)}{\omega_{ \pm}}\right]
\end{aligned}
$$

Note that when $\tau \rightarrow 0, x \rightarrow 0, \omega_{+} \rightarrow+\infty, \omega_{-} \rightarrow k \gamma_{f}$, and furthermore, $x \sqrt{a_{1}^{2}-4 a_{2}} \rightarrow 2 k \gamma_{c}$, $x a_{1} \rightarrow 2 k \gamma_{c}, x \omega_{+} \rightarrow 0$ and $\theta_{-} \rightarrow 2 \pi$. Thus, $\lim _{\tau \rightarrow 0} d S_{n}^{+}(\tau) / d \tau=+\infty$ and

$$
\lim _{\tau \rightarrow 0} \frac{d S_{n}^{-}(\tau)}{d \tau}=1+e^{-k \tau} \frac{\left(k+\gamma_{f}-\gamma_{c}\right)^{2}}{2 \gamma_{c}}\left(\frac{(n+1) \pi}{\sqrt{k \gamma_{f}}}+\frac{2}{k+\gamma_{f}-\gamma_{c}}\right) .
$$


Proof of Lemmas 3.8 and 3.9: $\quad$ Note that $S_{0}(\widetilde{\tau})=\widetilde{\tau}-\frac{\widetilde{\theta}}{\widetilde{\omega}}$, where $\widetilde{\omega}^{2}=\omega^{2}(\widetilde{\tau})=k \gamma_{f}+\mid k+$ $\gamma_{f}-\gamma_{c} \mid \sqrt{k \gamma_{f}}$ and

$$
\tilde{\theta}=\theta(\widetilde{\tau})= \begin{cases}2 \pi-\arccos \left(\frac{k+\gamma_{f}-\gamma_{c}}{k+\gamma_{f}-\gamma_{c}+2 \sqrt{k \gamma_{f}}}\right), & k+\gamma_{f}-\gamma_{c}>0, \\ \arccos \left(\frac{k+\gamma_{f}-\gamma_{c}}{k+\gamma_{f}-\gamma_{c}-2 \sqrt{k \gamma_{f}}}\right), & k+\gamma_{f}-\gamma_{c}<0 .\end{cases}
$$

This implies that $S_{0}(\widetilde{\tau})$ is determined by $k, \gamma_{f}$ and $\gamma_{c}$. Especially, when $k+\gamma_{f}-\gamma_{c} \rightarrow 0$, $S_{0}(\widetilde{\tau}) \rightarrow \infty$. The results follow by considering either $k \rightarrow 0$ or $\gamma_{f}=\gamma_{c}$.

\section{References}

Allen, H. and Taylor, M. (1990), 'Charts, noise and fundamentals in the london foreign exchange market', Economic Journal 100, 49-59.

Anufriev, M. and Dindo, P. (2009), 'Wealth-driven selection in a financial market with heterogeneous agents', Journal of Economic Behavior and Organization in presss.

Asada, T. and Yoshida, H. (2001), 'Stability, instability and complex behavior in macroeconomic models with policy lag', Discrete Dynamics in Nature and Society $\mathbf{5}, 281-$ 295.

Balasko, Y. and Royer, D. (1996), 'Stability of competitive equilibrium with respect to recursive and learning processes', Journal of Economic Theory 68, 319-348.

Beja, A. and Goldman, M. (1980), 'On the dynamic behavior of prices in disequilibrium', Journal of Finance 35, 235-247.

Beretta, E., Bischi, G. and Solimano, F. (1988), 'Oscillations in a system with material cycling', Journal of Mathematical Biology 26, 143-167.

Beretta, E. and Kuang, Y. (2002), 'Geometric stability switch criteria in delay differential systems with delay dependant parameters', SIAM Journal of Mathematical Analysis 33(5), 1144-1165.

Boswijk, H., Griffioen, G. and Hommes, C. (2000), Success and failure of technical trading strategies in the cocoa futures markets, CeNDEF working paper 00-06, University of Amsterdam.

Bradfield, J. (1979), 'A formal dynamic model of market making', Journal of Financial and Quantitative Analysis 14(2), 275-291.

Brock, H. and Hommes, C. (1998), 'Heterogeneous beliefs and routes to chaos in a simple asset pricing model', Journal of Economic Dynamics and Control 22, 1235-1274. 
Brock, W., Lakonishok, J. and LeBaron, B. (1992), 'Simple technical trading volatility and the stochastic properties of stock returns', Journal of Finance 47, 1731-1764.

Chiarella, C., Dieci, R. and He, X. (2009), Heterogeneity, Market Mechanisms and Asset Price Dynamics, Elsevier, pp. 277-344. in Handbook of Financial Markets: Dynamics and Evolution, Eds. Hens, T. and K.R. Schenk-Hoppe.

Chiarella, C. and He, X. (2001), 'Asset price and wealth dynamics under Heterogeneous expectations', Quantitative Finance 1, 509-526.

Chiarella, C. and He, X. (2002), 'Heterogeneous beliefs, risk and learning in a simple asset pricing model', Computational Economics 19, 95-132.

Chiarella, C. and He, X. (2003), 'Heterogeneous beliefs, risk and learning in a simple asset pricing model with a market maker', Macroeconomic Dynamics 7, 503-536.

Chiarella, C., He, X. and Hommes, C. (2006), 'A dynamic analysis of moving average rules', Journal of Economic Dynamics and Control 30, 1729-1753.

Chiarella, C., He, X., Hung, H. and Zhu, P. (2006), 'An analysis of the cobweb model with boundedly rational heterogeneous producers', Journal of Economic Behavior and Organization 61, 750-768.

Day, R. and Huang, W. (1990), 'Bulls, bears and market sheep', Journal of Economic Behavior and Organization 14, 299-329.

Dieci, R., Foroni, I., Gardini, L. and He, X. (2006), 'Market mood, adaptive beliefs and asset price dynamics', Chaos, Solitons and Fractals 29, 520-534.

Fama, E. (1970), 'Efficient capital markets: A review of theory and empirical eork', Journal of Finance 25, 383-423.

Fernandez-Rodriguez, F., Gonzalez-Martel, C. and Sosvilla-Rivero, S. (2000), 'On the profitability of technical trading rules based on artificial neural networks: Evidence from the madrid stock market', Economics Letters 69, 89-94.

Frankel, J. and Froot, K. (1986), 'Understanding the US dollars in the Eighties: The expectations of chartists and fundamentalists', Economic Record, Supplementary Issue 62, 24-38.

Frankel, J. and Froot, K. (1990), Chartists, Fundamentalists and the Demand for Dollars, Oxford University Press, New York, pp. 73-126. in Private Behaviour and Government Policy in Interdependent Economies, Eds. Courakis, A.S. and M.P. Taylor.

Gencay, R. (1998), 'Optimization of technical trading strategies and the profitability in security markets', Economics Letters 59, 249-254. 
Goldbaum, D. (2003), 'Profitable technical trading rules as a source of price instability', Quantitative Finance 3, 220-229.

Goodwin, R. (1951), 'The nonlinear accelerator and the persistence of business cycles', Econometrica 19, 1-17.

Gopalsamy, K. (1992), Stability and oscillations in delay differential equation of population dynamics, Kluwer Academic Publisher, London.

Haldane, J. B. S. (1932), 'A contribution to the theory of price fluctuations', Review of Economic Studies 1, 186-195.

Hale, J. and Kocak, H. (1991), Dynamics and Bifurcations, Vol. 3 of Texts in Applied Mathematics, Springer, New York.

He, X. (1998), 'The Lyapunov functionals for delay Lotka-Volterra type models', SIAM Journal of Applied Mathematics 58, 1222-1236.

He, X., Li, K., Wei, J. and Zheng, M. (2009), Market stability switches in continuous-time financial market with heterogeneous beliefs, Technical Report 252, Quantitative Finance Research Centre, University of Technology, Sydney.

He, X. and Li, Y. (2007), 'Power law behaviour, heterogeneity, and trend chasing', Journal of Economic Dynamics and Control 31, 3396-3426.

He, X. and Li, Y. (2008), 'Heterogeneity, convergence and autocorrelations', Quantitative Finance 8, 58-79.

Hirshleifer, D. (2001), 'Investor psychology and asset pricing', Journal of Finance 64, 1533-1597.

Hommes, C. (2006), 23 Heterogeneous Agent Models in Economics and Finance, Vol. 2 of Handbook of Computational Economics, North-Holland, pp. 1109-1186. in Agentbased Computational Economics, Eds. Tesfatsion, L. and K.L. Judd.

Howroyd, T. D. and Russell, A. M. (1984), 'Cournol oligopoly models with time delays', Journal of Mathematical Economics 13, 97-103.

Kalecki, M. (1935), 'A macroeconomic theory of the business cycle', Econometrica 3, 327344.

Küchler, U. and Platen, E. (2007), Time delay and noise explaining cyclical fluctuations in prices of commodities, Technical Report 195, Quantitative Finance Research Centre, University of Technology, Sydney.

Kuznetsov, Y. (2004), Elements of Applied Bifurcation Theory, SV, New York. 
Larson, A. (1964), 'The hog cycle as harmonic motion', Journal of Farm. Econ. 46, 375386.

LeBaron, B. (2006), Agent-based Computational Finance, Vol. 2 of Handbook of Computational Economics, North-Holland, pp. 1187-1233. in Agent-based Computational Economics, Eds. Tesfatsion, L. and K.L. Judd.

Levy, M., Levy, H. and Solomon, S. (2000), Microscopic Simulation of Financial Markets, from Investor Behavior to Market Phenomena, Academic Press, Sydney.

Lo, A., Mamaysky, H. and Wang, J. (2000), 'Foundations of technical analysis: Computational algorithms, statistical inference, and empirical implementation', Journal of Finance 55, 1705-1770.

Lux, T. (2004), Financial Power Laws: Empirical Evidence, Models and Mechanisms, Cambridge University Press. in Power Laws in the Social Sciences: Discovering Complexity and Non-equilibrium in the Social Universe, Eds. C. Cioffi.

Lux, T. (2009), Stochastic Behavioural Asset Pricing and Stylized Facts, Elsevier, pp. 161-215. in Handbook of Financial Markets: Dynamics and Evolution, Eds. Hens, T. and K.R. Schenk-Hoppe.

MacDonald, N. (1978), Time Lags in Biological Systems, Vol. 27 of Lecture Notes in Biomathematics, Springer, London.

Mackey, M. (1989), 'Commodity price fluctuations: price dependent delays and nonlinearities as explanatory factors', Journal of Economic Theory 48, 495-509.

Neely, C., Weller, P. and Dittmar, R. (1997), 'Is technical analysis in the foreign exchange market profitable? A genetic programming approach', Journal of Quantitative and Financial Analysis 32, 405-426.

Pesaran, M. and Timmermann, A. (1994), 'Forecasting stock returns, an examination of stock market trading in the presence of transaction costs', Journal of Forecasting 13, 335-367.

Pesaran, M. and Timmermann, A. (1995), 'Predictability of stock returns: Robustness and economic significance', Journal of Finance 50, 1201-1228.

Phillips, A. (1954), 'Stabilization policy in a closed economy', Economic Journal 64, 290321.

Phillips, A. (1957), 'Stabilization policy and time forms of lagged responsesin a closed economy', Economic Journal 67, 265-277.

Taylor, M. and Allen, H. (1992), 'The use of technical analysis in the foreign exchange market', Journal of International Money and Finance 11, 304-314. 
Yoshida, H. and Asada, T. (2007), 'Dynamic analysis of policy lag in a Keynes-Goodwin model: stability, instability, cycles and chaos', Journal of Economic Behavior and Organization 62, 441-469.

Zhu, M., Chiarella, C., He, X. and Wang, D. (2009), 'Does the market maker stabilize the market?', Physica A 388, 3164-3180.

Zschischang, E. and Lux, T. (2001), 'Some new results on the Levy, Levy and Solomon microscopic stock market model', Physica A 291, 563-573. 